\title{
A GENERAL HAMILTON-JACOBI FRAMEWORK FOR NON-LINEAR STATE-CONSTRAINED CONTROL PROBLEMS *
}

\author{
Albert Altarovici $^{1}$, Olivier Bokanowski ${ }^{1,2}$ and Hasnaa Zidani ${ }^{1}$
}

\begin{abstract}
The paper deals with deterministic optimal control problems with state constraints and non-linear dynamics. It is known for such problems that the value function is in general discontinuous and its characterization by means of a Hamilton-Jacobi equation requires some controllability assumptions involving the dynamics and the set of state constraints. Here, we first adopt the viability point of view and look at the value function as its epigraph. Then, we prove that this epigraph can always be described by an auxiliary optimal control problem free of state constraints, and for which the value function is Lipschitz continuous and can be characterized, without any additional assumptions, as the unique viscosity solution of a Hamilton-Jacobi equation. The idea introduced in this paper bypasses the regularity issues on the value function of the constrained control problem and leads to a constructive way to compute its epigraph by a large panel of numerical schemes. Our approach can be extended to more general control problems. We study in this paper the extension to the infinite horizon problem as well as for the two-player game setting. Finally, an illustrative numerical example is given to show the relevance of the approach.
\end{abstract}

Mathematics Subject Classification. 35B37, 49J15, 49Lxx, 49J45, 90C39.

Received December 19, 2011. Revised March 27, 2012.

Published online June 21, 2012.

\section{INTRODUCTION}

This paper deals with the characterization of the value function of a deterministic optimal control problem with state constraints. For a given finite horizon $T>0$, consider the dynamical system

$$
\begin{aligned}
& \dot{y}(s)=f(s, y(s), \alpha(s)), \text { a.e. } s \in(t, T), \\
& y(t)=x .
\end{aligned}
$$

\footnotetext{
Keywords and phrases. State constraints, optimal control problems, nonlinear controlled systems, Hamilton-Jacobi equations, viscosity solutions.

* This work has been partially supported by the EU under the programme "FP7-PEOPLE-2010-ITN", SADCO project, GA number 264735 SADCO.

1 Projet Commands, INRIA Saclay \& ENSTA ParisTech, 32 Bd. Victor, 75739 Paris Cedex 15, France.

Albert.Altarovici@inria.fr; Hasnaa.Zidani@ensta.fr

2 Univ. Paris Diderot, Sorbonne Paris Cité, Laboratoire Jacques-Louis Lions, UMR 7598, UPMC, CNRS, 75205 Paris, France. boka@math.jussieu.fr
} 
where $\alpha:[0, T] \rightarrow \mathcal{A}$ is a measurable function and $\mathcal{A}$ is a compact set of $\mathbb{R}^{p}(p \geq 1)$, and $f:[0, T] \times \mathbb{R}^{d} \times \mathcal{A} \rightarrow \mathbb{R}^{d}$ is a continuous function (see Sect. 2 for precise assumptions). The corresponding absolutely continuous solution will be denoted by $y=y_{t, x}^{\alpha}$.

For a given non-empty and closed subset $\mathcal{K}$ of $\mathbb{R}^{d}$, we consider a control problem and its value function given by

$$
\vartheta(t, x):=\min _{\alpha \in L^{\infty}((t, T), \mathcal{A})}\left\{\int_{t}^{T} \ell\left(s, y_{t, x}^{\alpha}(s), \alpha(s)\right) \mathrm{d} s+\varphi\left(y_{t, x}^{\alpha}(T)\right) \mid y_{t, x}^{\alpha}(\theta) \in \mathcal{K} \quad \forall \theta \in[t, T]\right\},
$$

with the usual convention that $\inf \emptyset=+\infty$, and where $\ell:[0, T] \times \mathbb{R}^{d} \times \mathcal{A} \rightarrow \mathbb{R}$ and $\varphi: \mathbb{R}^{d} \rightarrow \mathbb{R}$ are continuous functions (see Sect. 2 for precise assumptions). Without any further assumption, it may happen that the value function is discontinuous.

In the case when $\mathcal{K}=\mathbb{R}^{d}$ and $\varphi$ is Lipschitz continuous, under classical assumptions on $f$ and $\ell$, it is known that the value function $\vartheta$ is the unique continuous viscosity solution of a Hamilton-Jacobi equation [6, 8, 24]. This result is extended also to the lower semicontinuous (l.s.c.) setting in $[12,26]$.

When the control problem is in presence of state constraints $\left(\mathcal{K} \neq \mathbb{R}^{d}\right)$ a state-space constrained HJB equation has been associated to the value function $(1.2)$ in $[39,40]$. In our setting, this HJB equation takes the form:

$$
\begin{aligned}
& -u_{t}+\mathcal{H}\left(t, x, \nabla_{x} u\right)=0 \quad \text { in }(0, T) \times \mathcal{K}, \\
& u(T, x)=\varphi(x) \text { in } \mathcal{K},
\end{aligned}
$$

where $\mathcal{H}(t, x, p):=\max _{a \in \mathcal{A}}(-f(t, x, a) \cdot p-\ell(t, x, a))$. In Soner's formulation, a function $u$ is a viscosity solution of (1.3) if it is sub-solution in $(0, T) \times \stackrel{\circ}{\mathcal{K}}$ and a super-solution on $(0, T) \times \mathcal{K}$.

Even though it is easy to establish that the value function $\vartheta$ satisfies (1.3) in the constrained viscosity sense, it is more complicate to prove the uniqueness of the solution of (1.3). Actually, the uniqueness requires restrictive controllability assumptions on $\mathcal{K}$ and on the dynamics. The main difficulty comes from the fact that the state-space HJB equation may admit several solutions (in the constrained viscosity sense) if the behavior of the solution on the boundary is not taken into account, see $[16,31]$.

The most classical controllability assumption is called "inward pointing qualification condition (IPQ)". It was first introduced by Soner in $[39,40]$. It asks that at each point of the boundary of $\mathcal{K}$ there exists a field of the system pointing inward $\mathcal{K}$. Under this assumption the value function is Lipschitz continuous in $\mathcal{K}$, and uniqueness can be established. From the viewpoint of the dynamical system, the inward condition ensures that all the trajectory hitting the boundary can be approximated by a sequence of trajectories that stay inside the interior of $\mathcal{K}[29]$. We refer to $[18,35,36]$ for weaker inward pointing assumptions, and to $[32,33]$ for more properties and numerical approximation of continuous constrained viscosity solutions.

Another controllability assumption, called "outward pointing condition" (OPQ), has been considered in $[27,29]$. This assumption states that each point on the boundary of $\mathcal{K}$ can be reached by a trajectory coming from the interior of $\mathcal{K}$. Under this assumption it is still possible to characterize the value function as the unique lower semi-continuous solution of an HJB equation.

However there are many control problems where the controllability assumptions are never satisfied.

On the other hand, using the viability tools $[1,4]$ and non-smooth analysis it is always possible to characterize the value function and more precisely its epigraph, see $[2,5,19,20]$ and the references therein.

In the present work we show that the epigraph of $\vartheta$ can be described by using the knowledge of a Lipschitz continuous function, which turns out to be the value function of an auxiliary control problem free of state constraints. Then standard viscosity theory can be applied to characterize the new value function as the unique continuous viscosity solution of a variational HJ equation. More precisely, our approach consists in considering an auxiliary control problem defined by

$$
w^{g}(t, x, z):=\inf _{\alpha \in L^{\infty}((t, T), \mathcal{A})}\left(\int_{t}^{T} \ell\left(s, y_{t, x}^{\alpha}(s), \alpha(s)\right) \mathrm{d} s+\varphi\left(y_{t, x}^{\alpha}(T)\right)-z\right) \bigvee \max _{\theta \in(t, T)} g\left(y_{t, x}^{\alpha}(\theta)\right),
$$


where $a \bigvee b:=\max (a, b)$, and $g$ is a Lipschitz continuous function satisfying $g(x) \leq 0 \Leftrightarrow x \in \mathcal{K}$ (for instance the signed distance to $\mathcal{K}$ ). In this new problem there is no state constraints and the value function $w^{g}$ can be characterized as the unique continuous viscosity solution of the following HJ equation:

$$
\begin{aligned}
& \min \left(-\partial_{t} u+\max _{a \in \mathcal{A}}\left(-f(t, x, a) \nabla_{x} u+\ell(t, x, a) \partial_{z} u, \quad u-g(x)\right)=0, t \in[0, T),(x, z) \in \mathbb{R}^{d} \times \mathbb{R},\right. \\
& u(T, x, z)=(\varphi(x)-z) \vee g(x), \quad(x, z) \in \mathbb{R}^{d} \times \mathbb{R} .
\end{aligned}
$$

Moreover, under classical assumptions on $f, \ell$ and $\varphi$, the epigraph ${ }^{3}$ of $\vartheta$ satisfies $\mathcal{E} p i(\vartheta(t, \cdot))=\{(x, z) \in$ $\left.\mathbb{R}^{d} \times \mathbb{R}, w^{g}(t, x, z) \leq 0\right\}$, and we have $\vartheta(t, x)=\min \left\{z, w^{g}(t, x, z) \leq 0\right\}$.

Let us point out that a class of control problems with maximum costs has been already studied in [10,11]. Here, we use the term $\max _{\theta \in(t, T)} g\left(y_{t, x}^{\alpha}(\theta)\right)$ as an exact penalization of the state constraints. This kind of penalization has also already been successfully used to study the reachability analysis for some state constrained nonlinear systems, see [15,34]. Here, we use the exact penalization as a tool to bypass all the regularity issues in the characterization of $\vartheta$ and get a description of the epigraph without requiring any inward or outward pointing condition on the dynamics.

Our principal objective is to show that the exact penalization approach can be used in order to treat general optimal control problems with state constraints. We show that for each optimal control problem, corresponds an auxiliary control problem in with the state constraints are taken into account by an exact penalization. This simple idea can be extended to a large class of optimal control problems. In the present paper, we investigate the case of a finite horizon problem, the case of an infinite horizon control problem, and a two-player game problem. In each case, we show how the auxiliary control problem should be defined. The important property of the auxiliary control problem is to have a Lipschitz continuous value function, and that it can be characterized as the unique solution of a corresponding HJ equation. This approach needs to introduce a supplementary state variable, but it allows to have a proper way to characterize all the epigraph without controllability assumptions, and opens the way to a large class of numerical methods for computing this epigraph.

The paper is organized as follows. The setting of the problem and the assumptions are made precise in Section 2. Main results and proofs are presented in Section 3. The extension of the results to an infinite horizon control problem as well as two-player games is done in Sections 4, 5. Finally, a numerical example is given in Section 6 .

\section{Problem Formulation}

\subsection{Statement of the state-constrained control problem}

For a given non-empty compact subset $\mathcal{A}$ of $\mathbb{R}^{p}(p \geq 1)$ and a given $T \in \mathbb{R}(T>0)$, we consider the set of admissible controls defined by:

$$
\mathbb{A}:=\left\{\alpha:(0, T) \rightarrow \mathbb{R}^{p} \text { measurable, } \alpha(t) \in \mathcal{A} \text { a.e. }\right\} .
$$

Consider the controlled system:

$$
\begin{aligned}
& \dot{y}(s)=f(s, y(s), \alpha(s)), \text { a.e. } s \in(t, T), \\
& y(t)=x,
\end{aligned}
$$

\footnotetext{
${ }^{3}$ The epigraph at time $t$ is defined by $\mathcal{E} p i(\vartheta(t, \cdot)):=\left\{(x, z) \in \mathbb{R}^{d} \times \mathbb{R}, \vartheta(t, x) \leq z\right\}$.
} 
where $\alpha \in \mathbb{A}$, and $f:(0, T) \times \mathbb{R}^{d} \times \mathcal{A} \rightarrow \mathbb{R}^{d}$ is continuous and is assumed to satisfy the following regularity and growth properties:

(A1) $\left\{\begin{array}{l}(i) f:[0, T] \times \mathbb{R}^{d} \times \mathcal{A} \rightarrow \mathbb{R}^{d} \text { is continuous } \\ \text { (ii) There exists } L \geq 0, \text { for any } x, y \in \mathbb{R}^{d}, \text { for all } a \in \mathcal{A}, \text { and for all } t, s \in[0, T] \text { : } \\ |f(t, x, a)-f(s, y, a)| \leq L(|x-y|+|t-s|),\end{array}\right.$

where $|\cdot|$ is a norm on $\mathbb{R}^{d}$. It is known that under assumption (A1), for any $\alpha \in \mathbb{A}$ and for any $(t, x) \in(0, T) \times \mathbb{R}^{d}$, there exists a unique absolutely continuous trajectory $y=y_{t, x}^{\alpha}$ satisfying (2.1). The set of all feasible trajectories starting in $x$ at time $t$ will be denoted as:

$$
S_{[t, T]}(x):=\left\{y=y_{t, x}^{\alpha}, y \text { satisfies (2.1) for some measurable } \alpha \in \mathbb{A}\right\} .
$$

Under the assumption (A1), for any $t \in[0, T]$ and $x \in \mathbb{R}^{d}, S_{[t, T]}(x)$ is a compact subset of $W^{1,1}(t, T)$ for the topology of $C\left([0, T] ; \mathbb{R}^{d}\right)$. Moreover, the set-valued application $x \rightsquigarrow S_{[t, T]}(x)$ is Lipschitz continuous map from $\mathbb{R}^{d}$ in $C\left([0, T] ; \mathbb{R}^{d}\right)$.

Let $\mathcal{K}$ be a non-empty closed set of $\mathbb{R}^{d}$ (no additional assumptions on $\mathcal{K}$ will be made). A trajectory $y \in$ $S_{[t, T]}(x)$ will be said admissible (on the time interval $(t, T)$ ) if

$$
y(s) \in \mathcal{K}, \quad \text { for all } s \in(t, T) .
$$

Now, consider a distributed cost function $\ell:[0, T] \times \mathbb{R}^{d} \times \mathcal{A} \rightarrow \mathbb{R}$, satisfying:

(A2) $\left\{\begin{array}{l}(i) \ell:[0, T] \times \mathbb{R}^{d} \times \mathcal{A} \rightarrow \mathbb{R} \text { is continuous } \\ \text { (ii) There exists } L \geq 0, \text { for any } x, y \in \mathbb{R}^{d} \text {, for any } a \in \mathcal{A} \text { and for any } \forall t, s \in[0, T], \\ |\ell(t, x, a)-\ell(s, y, a)| \leq L(|x-y|+|t-s|),\end{array}\right.$

and a given final cost function $\varphi$ satisfying:

(A3) $\varphi: \mathbb{R}^{d} \rightarrow \mathbb{R}$ is Lipschitz continuous.

The state-constrained Bolza problem is formulated as follows:

$$
\vartheta(t, x):=\inf \left\{\int_{t}^{T} \ell\left(s, y_{t, x}^{\alpha}(s), \alpha(s)\right) \mathrm{d} s+\varphi\left(y_{t, x}^{\alpha}(T)\right) \mid \alpha \in \mathbb{A}, \quad \text { and } y_{t, x}^{\alpha}(s) \in \mathcal{K}, \forall s \in[t, T]\right\},
$$

with the convention that $\inf \emptyset=+\infty$.

First, let us recall that the Bolza problem (2.3) can be recast as a Mayer problem. For this, we introduce the following "augmented" dynamics $\hat{f}$ defined by

$$
\hat{f}(t,(x, z), a):=\left(\begin{array}{c}
f(t, x, a) \\
-\ell(t, x, a)
\end{array}\right) \quad \forall(x, z) \in \mathbb{R}^{d} \times \mathbb{R}, \forall a \in \mathcal{A}, \forall t \in(0, T)
$$

(the choice of $-\ell$ instead of $\ell$ is just for notational convenience). Introduce also, for $\alpha \in \mathbb{A}$, the augmented trajectory $\hat{y}=\hat{y}_{t,(x, z)}^{\alpha}$ solution of

$$
\begin{aligned}
& \dot{\hat{y}}(s)=\hat{f}(s, \hat{y}(s), \alpha(s)), s \in(t, T), \\
& \hat{y}(t)=(x, z)^{\top} .
\end{aligned}
$$

In particular, for any $\hat{x}=(x, z) \in \mathbb{R}^{d} \times \mathbb{R}$, the solution of (2.4) is given by: $\hat{y}_{t, \hat{x}}^{\alpha}(s)=\left(y_{t, x}^{\alpha}(s), \zeta_{t, \hat{x}}^{\alpha}(s)\right)$, where $\zeta_{t, \hat{x}}^{\alpha}(s):=z-\int_{t}^{s} \ell\left(\theta, y_{t, x}^{\alpha}(\theta), \alpha(\theta)\right) \mathrm{d} \theta$ and $y_{t, x}^{\alpha}$ is the solution of (2.1). Define the corresponding set of trajectories:

$$
\hat{S}_{[t, T]}(\hat{x}):=\left\{\hat{y}=\left(y_{t, x}^{\alpha}, \zeta_{t, \hat{x}}^{\alpha}\right), \hat{y} \text { satisfies }(2.4) \text { for some } \alpha \in \mathbb{A}\right\},
$$


for $\hat{x}=(x, z) \in \mathbb{R}^{d} \times \mathbb{R}$. The new control problem is then defined as follows:

$$
\hat{\vartheta}(t, \hat{x}):=\inf _{\hat{y}=(y, \zeta) \in \hat{S}_{[t, T]}(\hat{x})}\{\varphi(y(T))-\zeta(T), y(s) \in \mathcal{K}, \forall s \in(t, T)\} .
$$

It is clear that $\hat{\vartheta}(t, \hat{x})=\vartheta(t, x)-z$ for any $\hat{x}:=(x, z)$, and in particular $\vartheta(t, x)=\hat{\vartheta}(t,(x, 0))$. In the sequel, for simplicity of the presentation, the following assumption will be also assumed:

(A4) For any $(t, x) \in[0, T] \times \mathbb{R}^{d}, \hat{f}(t, x, \mathcal{A})$ is a convex set.

Therefore, for any $\hat{x} \in \mathbb{R}^{d} \times \mathbb{R}, \hat{S}_{[t, T]}(\hat{x})$ is a compact set. Moreover, the value function $\vartheta$ (resp. $\hat{\vartheta}$ ) is l.s.c. in $[0, T] \times \mathbb{R}^{d}$ (resp. in $\left.[0, T] \times \mathbb{R}^{d} \times \mathbb{R}\right)$.

Remark 2.1. Notice that $\hat{S}_{[t, T]}(\hat{x})$ may not be a closed set if (A4) is not satisfied, and therefore the infimum value of $\hat{\vartheta}$ may not be achieved by an admissible trajectory. In this case, it would be natural to consider the closure of $\hat{S}_{[t, T]}(\hat{x})$ (for the topology induced by the $C^{0}([t, T])$ norm), see [13,28]. For this, one should introduce $\hat{f}^{\sharp}$ the convexified set-valued dynamics

$$
\hat{f}^{\sharp}(t, \hat{x}):=\overline{\operatorname{co}(\hat{f}(t, \hat{x}, \mathcal{A}))}, \quad \text { for } t \in[0, T], \hat{x} \in \mathbb{R}^{d} \times \mathbb{R} .
$$

Here, since $\mathcal{A}$ is a compact set of $\mathbb{R}^{d}$ and $\hat{f}$ is continuous, by the Carathéodory theorem $\operatorname{co}(\hat{f}(t, \hat{x}, \mathcal{A}))$ is also a compact set and thus $\hat{f}^{\sharp}(t, \hat{x}) \equiv \operatorname{co}(\hat{f}(t, \hat{x}, \mathcal{A}))$. Under assumptions (A1)-(A2) the following differential inclusion admits absolutely continuous solutions in $[t, T]$ (see [3]):

$$
\begin{aligned}
& \dot{y}(s) \in \hat{f}^{\sharp}(s, y(s)), \quad \text { a.e. } s \in(t, T), \\
& y(t)=x .
\end{aligned}
$$

Let us denote by $\hat{S}_{[t, T]}^{\sharp}(x, z)$ the set of all the solutions of $(2.6)$. This set is precisely the closure of $\hat{S}_{[t, T]}^{\sharp}(x, z)$ for the topology of $C^{0}(0, T)$ (see for instance [28]):

$$
\hat{S}_{[t, T]}^{\sharp}(\xi) \equiv{\overline{\hat{S}_{[t, T]}(x, z)}}^{C^{0}}
$$

Moreover, by Filippov's theorem, $\hat{S}_{[t, T]}^{\sharp}(x, z)$ is a compact set of $C^{0}([t, T])$. Now, define the relaxed control problem, and its associated value function $\vartheta^{\sharp}$, as follows:

$$
\vartheta^{\sharp}(t, \hat{x}):=\min _{\hat{y}=(y, \zeta) \in \hat{S}_{[t, T]}^{\sharp}(\hat{x})}\{\varphi(y(T))-\zeta(T) \mid y(s) \in \mathcal{K}, \forall s \in(t, T)\}
$$

where $\hat{y}(s)=(y(s), \zeta(s))$ denotes the two components of a given trajectory of $\hat{S}_{[t, T]}^{\sharp}(x, z)$. In this case, the function $\vartheta^{\sharp}$ is l.s.c. and the minimum in $(2.8)$ is achieved.

\subsection{Some properties of the value function $\hat{\vartheta}$}

In order to state the dynamic programming principle (DPP), two more notations will be used: the set of admissible trajectories starting from $\hat{x}$

$$
\hat{S}_{[t, T]}^{\mathcal{K}}(\hat{x}):=\left\{\hat{y} \in \hat{S}_{[t, T]}(\hat{x}), \hat{y}(s) \in \mathcal{K} \times \mathbb{R} \text { for } s \in[t, T]\right\},
$$

and the set of admissible backward trajectories arriving at $\hat{x}$

$$
\begin{aligned}
\hat{S}_{[\tau, t]}^{\mathcal{K},-}(\hat{x}):= & \left\{\hat{y} \in W^{1,1}(\tau, t), \hat{y} \text { satisfies }(2.4 \mathrm{a}) \text { for some measurable } \alpha \in \mathbb{A} \text { on }[\tau, t],\right. \\
& \hat{y}(t)=x \text { and } \hat{y}(s) \in \mathcal{K} \times \mathbb{R} \text { for } s \in[\tau, t]\} .
\end{aligned}
$$


Lemma 2.2. Assume (A1)-(A4). For any $\hat{x} \in \mathbb{R}^{d} \times \mathbb{R}$ and any $t \in[0, T[$, the following statements hold:

(i) (Forward DPP) For any $h>0$ such that $t+h \leq T$ :

$$
\hat{\vartheta}(t, \hat{x})=\min _{\hat{y} \in \hat{S}_{[t, t+h]}^{\kappa}(\hat{x})} \hat{\vartheta}(t+h, \hat{y}(t+h))
$$

with the convention $\hat{\vartheta}(t, \hat{x})=+\infty$ whenever $\hat{S}_{[t, t+h]}^{\mathcal{K}}(\hat{x})=\emptyset$.

(ii) (Backward DPP) For all $s \in[0, t]$ and $\hat{x} \in \mathbb{R}^{d} \times \mathbb{R}$, for every $\hat{y} \in \hat{S}_{[t-h, t]}^{\mathcal{K},-}(\hat{x})$ :

$$
\hat{\vartheta}(t, \hat{x}) \geq \hat{\vartheta}(t-h, \hat{y}(t-h)) .
$$

Since $\hat{S}_{[t, T]}(\hat{x})$ is a compact set, the forward DPP leads to the statement that $\hat{\vartheta}$ satisfies the increasing principle, i.e. for any $(t, \hat{x}) \in[0, T] \times \mathbb{R}^{d} \times \mathbb{R}$ :

$$
\exists \hat{y} \in \hat{S}_{[t, T]}(\hat{x}), \quad \hat{\vartheta}(t, \hat{x}) \geq \hat{\vartheta}(t+s, \hat{y}(t+s)) \quad \text { for any } s \in[t, T-t] .
$$

This amounts to saying that the epigraph of $\hat{\vartheta}$ is weakly invariant with respect to the set-valued application:

$$
(t, \hat{x}) \rightsquigarrow(1, \hat{f}(t, \hat{x}, \mathcal{A}), 0)^{\top} .
$$

Under assumption (A1)-(A4), the mapping $(t, x) \rightsquigarrow \hat{f}(t, \hat{x}, \mathcal{A})$ is upper semi-continuous and has nonempty convex compact images. Therefore, the characterization of the weak invariance principle by means of a HJB inequality is straightforward. More precisely, one can prove that (see $[9,16,22,26])$ :

$$
u \text { satisfies }(2.9) \Longleftrightarrow-\partial_{t} u(t, \hat{x})+\sup _{a \in \mathcal{A}}\left(-\hat{f}(t, \hat{x}, a) \cdot D_{\hat{x}} u(t, \hat{x})\right) \leq 0 \quad \operatorname{in}[0, T] \times \mathcal{K} \times \mathbb{R} .
$$

On the other hand, the backward DPP expresses the fact that $\hat{\vartheta}$ satisfies also the decreasing principle of the value function $\hat{\vartheta}$ along admissible backward trajectories $\hat{y} \in \hat{S}_{[t-s, t]}^{\mathcal{K},-}(\hat{x})$ for every $\hat{x} \in \mathcal{K} \times \mathbb{R}$ and every $t \in[0, T]$ and $s \in[0, t]$.

Let $T_{\mathcal{K}}(\hat{x})$ denote the tangent cone of $\mathcal{K}$ at $\hat{x}$. If we assume that $\mathcal{K}$ is smooth enough and has a nonempty interior, and if the vector-field $\hat{f}(t, \hat{x}, \mathcal{A})$ is assumed to satisfy $\hat{f}(t, \hat{x}, \mathcal{A}) \cap T_{\mathcal{K}}(\hat{x}) \neq \emptyset$, then from the decreasing principle one can conclude that $\mathcal{E} p i(\hat{\vartheta})$ is strongly invariant with respect to the application

$$
(t, \hat{x}) \rightsquigarrow\left[\begin{array}{c}
-1 \\
-\left(\hat{f}(t, \hat{x}, \mathcal{A}) \cap T_{\mathcal{K}}(\hat{x})\right) \\
0
\end{array}\right] .
$$

In general, although $\hat{f}$ is Lipschitz continuous, the set-valued application $(t, \hat{x}) \rightsquigarrow \hat{f}(t, \hat{x}, \mathcal{A}) \cap T_{\mathcal{K}}(\hat{x})$ may have empty images and may not be Lipschitz continuous. Therefore, the characterization of the strong invariance principle by means of a HJB inequality is not clear (see [16,22]). As mentioned in the introduction, several works have been done to investigate the characterization of strong invariance under additional controllability assumptions.

In the next section, we will follow a completely different reasoning and will prove that the epigraph of $\vartheta$ can be described using a value function of an auxiliary control problem without state-constraints. This description does not require any additional assumptions on $\mathcal{K}$ or the regularity of $\vartheta$.

\section{Characterization of the epigraph}

\subsection{Auxiliary control problem. Main result}

Here we shall focus on the characterization of the epigraph of $\vartheta(t,$.

$$
\mathcal{E} \operatorname{pi}(\vartheta(t, .)):=\left\{(x, z) \in \mathbb{R}^{d} \times \mathbb{R}, \vartheta(t, x) \leq z\right\} .
$$


(This set also corresponds to $\left\{\hat{x}=(x, z) \in \mathbb{R}^{d} \times \mathbb{R}, \hat{\vartheta}(t, \hat{x}) \leq 0\right\}$.) For this, consider a "level set" function $g: \mathbb{R}^{d} \rightarrow \mathbb{R}$, Lipschitz continuous, that represents the set of constraints $\mathcal{K}$ in the following way:

$$
\forall x \in \mathbb{R}^{d}, \quad g(x) \leq 0 \Leftrightarrow x \in \mathcal{K} .
$$

Since $\mathcal{K}$ is closed, such a function $g$ exists. Indeed, if we denote by $d_{K}$ the signed distance to $\mathcal{K}$ (where $d_{\mathcal{K}}(x):=$ $d(x, \mathcal{K})$ if $x \notin \mathcal{K}$ and $d_{\mathcal{K}}(x)=-d\left(x, \mathbb{R}^{d} \backslash \mathcal{K}\right)$ otherwise), then the function $g \equiv d_{\mathcal{K}}(\cdot)$ is Lipschitz continuous and satisfies the statement (3.1). Therefore, for any $y \in S_{[t, T]}(x)$ :

$$
(y(\theta) \in \mathcal{K}, \forall \theta \in[t, T]) \Leftrightarrow \max _{\theta \in[t, T]} g(y(\theta)) \leq 0 .
$$

Now, introduce the auxiliary control problem and its associated value function $w^{g}$ defined by:

$$
w^{g}(t, x, z):=\inf _{\hat{y}=(y, \zeta) \in \hat{S}[t, T]}(x, z) \quad(\varphi(y(T))-\zeta(T)) \bigvee \max _{\theta \in(t, T)} g(y(\theta))
$$

for $x \in \mathbb{R}^{d}, z \in \mathbb{R}, t \in[0, T]$, and where we have used, for convenience, the notation $a \vee b:=\max (a, b)$.

In this auxiliary control problem, the term " $\max _{\theta \in[t, T]} g(y(\theta))$ " is an exact penalization of the state constraints. Here, we shall use the problem (3.2) to characterize the epigraph of the value function $\vartheta$ without requiring any additional assumptions on $\mathcal{K}$ or on the dynamics $\hat{f}$.

Theorem 3.1. Assume that (A1)-(A4) hold and that $\mathcal{K}$ is closed and non-empty. Then for any $t \in[0, T]$ and $(x, z) \in \mathbb{R}^{d} \times \mathbb{R}$, the following holds:

$$
\vartheta(t, x)-z \leq 0 \quad \Longleftrightarrow \hat{\vartheta}(t,(x, z)) \leq 0 \quad \Longleftrightarrow \quad w^{g}(t, x, z) \leq 0 ;
$$

(ii) moreover, the function $\vartheta$ is characterized by $w^{g}$ through the following relation

$$
\vartheta(t, x)=\min \left\{z \in \mathbb{R}, w^{g}(t, x, z) \leq 0\right\} .
$$

Proof. (i) Let us assume that $\vartheta(t, x) \leq z$. There exists a sequence $y_{n}$ of admissible trajectories of $S_{[t, T]}(x)$, such that

$$
\lim _{n \rightarrow \infty} \int_{t}^{T} \ell\left(s, y_{n}(s), \alpha(s)\right) \mathrm{d} s+\varphi\left(y_{n}(T)\right)-z=\vartheta(t, x)-z \leq 0 .
$$

Since all the trajectories $y_{n}$ are admissible, we have, for all $n \geq 0, \max _{\theta \in[t, T]} g\left(y_{n}(\theta)\right) \leq 0$. Hence

$$
\begin{aligned}
w^{g}(t, x, z) & \leq \liminf _{n \rightarrow \infty}\left(\int_{t}^{T} \ell\left(s, y_{n}(s), \alpha(s)\right) \mathrm{d} s+\varphi\left(y_{n}(T)\right)-z\right) \bigvee \max _{\theta \in[t, T]} g\left(y_{n}(\theta)\right) \\
& \leq 0 .
\end{aligned}
$$

Conversely, let us assume that $w^{g}(t, x, z) \leq 0$. We know that $\hat{S}_{[t, T]}(\xi)$ is a compact set in $C^{0}([t, T])$, hence the infimum in $w^{g}(t, x, z)$ is achieved by some trajectory $y \in \hat{S}[t, T]((x, z))$. Moreover,

$$
0 \geq w^{g}(t, x, z)=\left(\int_{t}^{T} \ell(s, y(s), \alpha(s)) \mathrm{d} s+\varphi(y(T))-z\right) \bigvee \max _{\theta \in[t, T]} g(y(\theta)) .
$$

Therefore, on one hand, $\max _{\theta \in[t, T]} g(y(\theta)) \leq 0$ and $y$ satisfies the state constraints, and on the other hand,

$$
\vartheta(t, x)-z \leq \int_{t}^{T} \ell(s, y(s), \alpha(s)) \mathrm{d} s+\varphi(y(T))-z \leq 0
$$

which is the desired result.

The proof of $(i i)$ is a consequence of $(i)$. 
Remark 3.2. When the convexity assumption (A4) is not satisfied, the statements of the above theorem are valid for the l.s.c. value function $\vartheta^{\sharp}$ instead of $\hat{\vartheta}$. Indeed, let $\ell \equiv 0, f(t, x, \alpha):=(1, \alpha)^{\top}$ with $\alpha \in \mathcal{A}:=\{ \pm 1\}$, $\mathcal{K}:=\left\{\left(x_{1}, x_{2}\right) \in \mathbb{R}^{2},\left|x_{2}\right| \leq\left|x_{1}-\frac{1}{2}\right|^{2}\right\}$, and $T=1$. One can check that, for $\bar{x}=(0,0)^{\top}$, there exist no admissible trajectories starting at $\bar{x}$ and staying in $\mathcal{K}$ on $[0, T]$. Hence $\hat{\vartheta}(0,(\bar{x}, z))=+\infty$ for all $z \in \mathbb{R}$. On the other hand, let $\left(y_{n}\right)_{n \geq 1}$ be the sequence of trajectories defined for $\theta \in(0, T)$ by

$$
y_{n}(\theta)= \begin{cases}\left(\frac{k}{n}, 0\right)^{\top}+\left(\theta-\frac{k}{n}\right)(1,1)^{\top} & \text { if } \theta \in\left[\frac{k}{n}, \frac{k+1 / 2}{n}[,\right. \\ \left(\frac{k+1 / 2}{n}, \frac{1 / 2}{n}\right)^{\top}+\left(\theta-\frac{k+1 / 2}{n}\right)(1,-1)^{\top} \text { if } \theta \in\left[\frac{k+1 / 2}{n}, \frac{k+1}{n}[.\right.\end{cases}
$$

Then $y_{n}$ converges uniformly on $[0,1]$ toward the limit $y(t)=(t, 0)^{\top}$, and $w^{g}(0, x, z)$ will have a finite negative value whenever $\varphi\left((T, 0)^{\top}\right)<z$.

\subsection{A particular case: backward reachable sets}

Consider the problem of backward reachable sets from a closed target $\mathcal{C}$ of $\mathbb{R}^{d}$. It consists in characterizing, for every $t \in[0, T]$, the set of all initial positions from which it is possible to find an admissible trajectory that reaches the target at time $T$ while lying in the set $\mathcal{K}$ on $[t, T]$ :

$$
\mathcal{R}(t):=\left\{x \in \mathbb{R}^{d}: \exists y \in S_{[t, T]}(x) \text { such that } y(T) \in \mathcal{C}, \text { and } y(s) \in \mathcal{K} \text { for } s \in[t, T]\right\} .
$$

In that case we can consider $\ell \equiv 0$ and a Lipschitz continuous function $\phi: \mathbb{R}^{d} \rightarrow \mathbb{R}$ satisfying:

$$
\forall x \in \mathbb{R}^{d}, \quad x \in \mathcal{C} \quad \Leftrightarrow \quad \phi(x) \leq 0 .
$$

As in [15], we consider the control problem:

$$
v^{g}(t, x)=\inf _{y \in S_{[t, T]}(x)} \phi(y(T)) \bigvee \max _{\theta \in(t, T)} g(y(\theta)) .
$$

Similar value functions have been also considered by Margellos and Lygeros in [34]. Therefore, under the same assumption (A1) and with a closed set $\mathcal{K}$, the backward reachable set is equivalent to:

$$
\mathcal{R}(t) \equiv\left\{x \in \mathbb{R}^{d} \mid v^{g}(t, x) \leq 0\right\},
$$

in other terms, the region where the function $v^{g}(t, \cdot)$ is non-positive is precisely the backward reachable set at time $t$, see [15]. In view of this result, Theorem 3.1 can be seen as a generalization to a more general setting where the function $\varphi$ is any Lipschitz continuous function. Actually, in the general case, the epigraph of the function $\varphi$ should be considered as the target set and the epigraph of $\hat{\vartheta}(t, \cdot)$ represents the backward reachable set under the dynamics $\hat{f}$. This claim can be proved by using viability tools, see for instance $[2,20]$. Moreover, taking this claim into account, one can compute the value function by using viability algorithm [5,38]. Here, we use only viscosity arguments and give a description of the epigraph by means of a simple continuous solution to a HJ equation. This opens the way to use a very large panel of numerical schemes (Semi-Lagrangian, ENO, WENO, Discontinuous Galerkin, ....).

\subsection{Properties of the auxiliary value function $w^{g}$}

The main feature of the auxiliary control problem (3.2) lies in the fact that this new problem is not under state constraints any more. Moreover, function $w^{g}$ enjoys more regularity properties and can be characterized by a Hamilton-Jacobi equation without making any controllability assumptions. Let us denote by $\mathcal{H}$ the Hamiltonian defined on $[0, T] \times \mathbb{R}^{d} \times \mathbb{R} \times \mathbb{R}^{d} \times \mathbb{R}$ by:

$$
\mathcal{H}(t, x, z, p, q):=\max _{a \in \mathcal{A}}(-f(t, x, a) \cdot p+\ell(t, x, a) \cdot q) .
$$


Proposition 3.3. Assume that (A1)-(A4) hold and let $g$ be a Lipschitz continuous function satisfying (3.1). Then $w^{g}$ is a locally Lipschitz continuous function on $[0, T] \times \mathbb{R}^{d} \times \mathbb{R}$. Furthermore, for any $t \in[0, T]$, and $h \geq 0$ such that $t+h \leq T$,

$$
w^{g}(t, x, z):=\min _{\hat{y}=(y, \zeta) \in \hat{S}_{[t, t+h]}(x, z)}\left\{w^{g}(t+h, y(t+h), \zeta(t+h)) \bigvee \max _{\theta \in(t, t+h)} g(y(\theta))\right\}
$$

Proof. The proof of the DPP (3.6) is classical and can be obtained by the same arguments as in [10,11]. Consider $\hat{x}=(x, z), \hat{x}^{\prime}=\left(x^{\prime}, z^{\prime}\right) \in \mathbb{R}^{d} \times \mathbb{R}, t \in[0, T]$, and $w_{0}(\hat{x}):=w^{g}(0, \hat{x}) \equiv \max (\varphi(x)-z, g(x))$. By using the definition of $w^{g}$ and the elementary inequalities:

$$
\max (A, B)-\max (C, D) \leq \max (A-C, B-D), \quad \text { and } \quad \inf A_{\alpha}-\inf B_{\alpha} \leq \sup \left(A_{\alpha}-B_{\alpha}\right)
$$

we get:

$$
\begin{aligned}
\left|w^{g}(t, \hat{x})-w^{g}\left(t, \hat{x}^{\prime}\right)\right| & \leq \sup _{\alpha \in \mathbb{A}} \max \left(\left|w_{0}\left(\hat{y}_{t, \hat{x}}^{\alpha}(T)\right)-w_{0}\left(\hat{y}_{t, \hat{x}^{\prime}}^{\alpha}(T)\right)\right|, \max _{\theta \in(t, T)}\left|g\left(y_{t, x}^{\alpha}(\theta)\right)-g\left(y_{t, x^{\prime}}^{\alpha}(\theta)\right)\right|\right), \\
& \leq \sup _{\alpha \in \mathbb{A}}\left(L_{0}\left|\hat{y}_{t, \hat{x}}^{\alpha}(T)-\hat{y}_{t, \hat{x}^{\prime}}^{\alpha}(T)\right|, L_{g} \max _{\theta \in(t, T)}\left|y_{t, x}^{\alpha}(\theta)-y_{t, x^{\prime}}^{\alpha}(\theta)\right|\right)
\end{aligned}
$$

where $L_{0}$ and $L_{g}$ denotes respectively the Lipschitz constant of $w_{0}$ and $g$. By assumption (A1), for $\theta \in(t, T)$, $\left|\hat{y}_{t, \hat{x}}^{\alpha}(\theta)-\hat{y}_{t, \hat{x}^{\prime}}^{\alpha}(\theta)\right| \leq \mathrm{e}^{\hat{L}(\theta-t)}\left|\hat{x}-\hat{x}^{\prime}\right| \leq \mathrm{e}^{\hat{L} T}\left|\hat{x}-\hat{x}^{\prime}\right|$ (where $\hat{L}$ is the Lipschitz constant of $\hat{f}$ ). Then we conclude that:

$$
\left|w^{g}(t, \hat{x})-w^{g}\left(t, \hat{x}^{\prime}\right)\right| \leq \max \left(L_{0}, L_{g}\right) \mathrm{e}^{\hat{L} T}\left|\hat{x}-\hat{x}^{\prime}\right|
$$

On the other hand, let $\hat{x}=(x, z)$ be in $\mathbb{R}^{d} \times \mathbb{R}$, and let $t \geq 0, h \geq 0$. Using that $w^{g}(t, \hat{x}) \geq g(x)$, we deduce from the dynamic programming principle for $w^{g}$ that

$$
\begin{aligned}
\left|w^{g}(t+h, \hat{x})-w^{g}(t, \hat{x})\right| & =\left|\inf _{\alpha} \max \left(w^{g}\left(t, \hat{y}_{t, \hat{x}}^{\alpha}(t+h)\right), \max _{\theta \in[t, t+h]} g\left(y_{t, x}^{\alpha}(\theta)\right)\right)-\max \left(w^{g}(t, \hat{x}), g(x)\right)\right| \\
& \left.\leq \sup _{\alpha} \max \left(\mid w^{g}\left(t, \hat{y}_{t, \hat{x}}^{\alpha}(t+h)\right)-w^{g}(t, \hat{x})\right)\left|, \max _{\theta \in(t, t+h)}\right| g\left(y_{t, x}^{\alpha}(\theta)\right)-g(x) \mid\right) \\
& \leq \max \left(\max \left(L_{0}, L_{g}\right) \mathrm{e}^{\hat{L} T}\left|\hat{y}_{t, \hat{x}}^{\alpha}(t+h)-\hat{x}\right|, L_{g} \max _{\theta \in(t, t+h)}\left|y_{t, x}^{\alpha}(\theta)-x\right|\right)
\end{aligned}
$$

where we have used (3.8). 
Furthermore, denoting $C_{f}:=\max _{(s, a) \in(0, T) \times \mathcal{A}}|f(s, 0, a)|<\infty$, we have $|f(s, x, a)| \leq C_{f}+L|x|$. Hence by a Gronwall estimate, we have $\left|y_{t, x}^{\alpha}(\theta)-x\right| \leq\left(C_{f}+L|x|\right) \mathrm{e}^{L h} h \leq\left(C_{f}+L|x|\right) \mathrm{e}^{L T} h$ for $\theta \in(t, t+h)$. We obtain in the same way the estimate: $\left|\hat{y}_{t, \hat{x}}^{\alpha}(\theta)-\hat{x}\right| \leq\left(\hat{C}_{f}+\hat{L}|\hat{x}|\right) \mathrm{e}^{\hat{L} T} h$ for every $\theta \in(t, t+h)$.

Therefore, we conclude that $\left|w_{g}\left(t^{\prime}, \hat{x}\right)-w_{g}(t, \hat{x})\right| \leq C(1+|\hat{x}|)\left|t^{\prime}-t\right|$ for some constant $C>0$. Combining all the inequalities above, we get:

$$
\left|w_{g}\left(t^{\prime}, \hat{x}^{\prime}\right)-w_{g}(t, \hat{x})\right| \leq C(1+|\hat{x}|)\left(\left|t^{\prime}-t\right|+\left|\hat{x}^{\prime}-\hat{x}\right|\right),
$$

for some constant $C \geq 0$. In particular the following linear growth holds: $\left|w_{g}(t, \hat{x})\right| \leq C(1+|\hat{x}|)$.

Moreover, the following proposition holds.

Proposition 3.4. Assume that (A1)-(A4) are satisfied. Then the function $u=w^{g}$ is the unique continuous viscosity solution of the following HJB equation

$$
\begin{aligned}
& \min \left(-\partial_{t} u(t, x, z)+\mathcal{H}\left(t, x, z, \nabla_{x} u, \partial_{z} u\right), \quad u(t, x, z)-g(x)\right)=0, \quad \forall t \in[0, T), x \in \mathbb{R}^{d}, z \in \mathbb{R}, \\
& u(T, x, z)=(\varphi(x)-z) \vee g(x), \quad x \in \mathbb{R}^{d}, \quad z \in \mathbb{R}
\end{aligned}
$$

Proof. The HJB equation can be derived from the DPP satisfied by $w^{g}$. For sake of completeness, we give here the main lines of the proof. We first show that $w^{g}$ is a solution of (3.9). The fact that $w^{g}$ satisfies the initial condition comes directly from the definition of $w^{g}$.

Let us check the super-solution property of $w^{g}$. From the DPP, we get that for any $\tau \geq 0$

$$
w^{g}(t,(x, z)) \geq \min _{\hat{y}=(y, \zeta) \in \hat{S}_{[t, t+h]}(x, z)} w^{g}(t+h, y(t+h), \zeta(t+h)) .
$$

Hence, classical arguments in viscosity theory yield to:

$$
-\partial_{t} w^{g}+\mathcal{H}\left(t, x, z, \nabla_{x} w^{g}, \partial_{z} w^{g}\right) \geq 0
$$

in the viscosity sense. Moreover, by definition of $w^{g}$, for every $(t, x, z) \in[0, T] \times \mathbb{R}^{d} \times \mathbb{R}$, we have

$$
w^{g}(t, x, z) \geq \min _{\hat{y}=(y, \zeta) \in \hat{S}_{[t, T]}(x, z)} \max _{\theta \in[t, T]} g(y(\theta)) \geq g(x) .
$$

Combining these two inequalities, we get

$$
\min \left(\partial_{t} w^{g}+\mathcal{H}\left(t, x, z, \nabla_{x} w^{g}, \partial_{z} w^{g}\right), w^{g}-g\right) \geq 0
$$

in the viscosity sense, i.e., $w^{g}$ is a super-solution of (3.2).

It remains to prove that $w^{g}$ is a sub-solution. Let $(x, z) \in \mathbb{R}^{d} \times \mathbb{R}$ and $t \in[0, T]$. If $w^{g}(t, x, z) \leq g(x)$, then it is clear that $w^{g}$ satisfies:

$$
\min \left(-\partial_{t} w^{g}+\mathcal{H}\left(t, x, z, \nabla_{x} w^{g}, \partial_{z} w^{g}\right), w^{g}(t, x, z)-g(x)\right) \leq 0 .
$$

Now, assume that $w^{g}(t, x, z)>g(x)$. By continuity of $g$ and $w^{g}$, there exists some $\tau>0$ such that for every $\hat{y}=(y, \zeta) \in \hat{S}_{[t, t+h]}(x, z)$, we have: $w^{g}(\theta, y(\theta), \zeta(\theta))>g(y(\theta))$ for all $\theta \in[t, t+h]$ (since $y(\theta)$ will stay in a neighborhood of $x$ ). Hence, by using the DPP, we get that

$$
w^{g}(t, x, z)=\min _{\hat{y}=(y, \zeta) \in \hat{S}_{[t, T]}(x, z)} w^{g}(t+h, y(t+h), \zeta(t+h)), \quad \text { for any } 0 \leq h \leq T-t .
$$


Therefore, with classical arguments [6] one can obtain that $\partial_{t} w^{g}(t, x, z)+\mathcal{H}\left(t, x, z, \nabla_{x} w^{g}, \partial_{z} w^{g}\right) \leq 0$ in the viscosity sense. Therefore, $w^{g}$ is a viscosity sub-solution of (3.2).

The fact that $w^{g}$ is the unique continuous viscosity solution of (3.2) follows from the general comparison principle in Appendix A, and the fact that there exists $C_{0}>0$ such that:

$$
\begin{aligned}
&\left|\mathcal{H}(t, x, z, p, q)-\mathcal{H}\left(s, x^{\prime}, z^{\prime}, p, q\right)\right| \leq C_{0}(|p|+|q|+1)\left(|t-s|+\left|x-x^{\prime}\right|+\left|z-z^{\prime}\right|\right), \\
&\left|\mathcal{H}(t, x, z, p, q)-\mathcal{H}\left(t, x, z, p^{\prime}, q^{\prime}\right)\right| \leq C_{0}(|x|+1)\left(\left|p-p^{\prime}\right|+\left|q-q^{\prime}\right|\right),
\end{aligned}
$$

for every $(x, z),\left(x^{\prime}, z^{\prime}\right) \in \mathbb{R}^{d} \times \mathbb{R}$ and for every $(p, q),\left(p^{\prime}, q^{\prime}\right) \in \mathbb{R}^{d} \times \mathbb{R}$.

Remark 3.5 (link with optimal stopping time). The auxiliary value function $w^{g}$ can be also interpreted as the value of an optimal stopping time problem, for $t \leq T$ :

$$
u(t, x, z):=\sup _{\tau \in[t, T]} \inf _{(y, \zeta) \in \hat{S}_{[t, T]}(x, z)} g(y(\tau)) 1_{\{\tau<T\}}+(\varphi(y(\tau))-\zeta(\tau)) \vee g(y(\tau)) 1_{\{\tau=T\}}
$$

(see for instance [6], Chap. III, Sect. 4.2). Indeed, the value function $u$ is continuous, and viscosity solution of the HJ equation (3.9). By the uniqueness of continuous viscosity solution of (3.9), we get that $u=w^{g}$. This interpretation of $w^{g}$ as an optimal value of a stopping-time problem can be also understood by remarking that:

$$
\max _{\tau \in[t, T]} g(y(\tau)) \bigvee(\varphi(y(\tau))-\eta(\tau))=\sup _{\tau \in[t, T]}\left(g(y(\tau)) 1_{\{\tau<T\}}+(\varphi(y(\tau))-\zeta(\tau)) \vee g(y(\tau)) 1_{\{\tau=T\}}\right) .
$$

Finally, let us stress that this equivalence between the penalized and the optimal-stopping problems does not mean that the original state-constrained problem itself has any interpretation as an optimal-stopping time. The only link is that when, for $(t, x, z) \in(0, T) \times \mathbb{R}^{d} \times \mathbb{R}$, the optimal-stoping time value $u(t, x, z)$ is negative, then $(t, x, z)$ belongs to the epigraph of $\vartheta$.

Remark 3.6. Notice that when $\mathcal{K}$ is a bounded set, then it is possible to modify $f, \ell$ and $g$ outside $\mathcal{K}$ in order to get a bounded auxiliary value function. Indeed, if $\mathcal{K} \subset B(0, R)$ then by setting $h(x):=d(x, B(0, R))$, it is possible to consider $f_{R}(t, x, \alpha):=f(t, x, \alpha)(1-h(x))_{+}, \ell_{R}(t, x, \alpha):=\ell(t, x, \alpha)(1-h(x))_{+}$and $g_{R}(x)=\max (g(x), R)$ (the functions $f_{R}, \ell_{R}$ and $g_{R}$ coincide respectively with $f, \ell$ and $g$ on $\mathcal{K}$ ). Moreover, $f_{R}, \ell_{R}$ and $g_{R}$ are still Lipschitz continuous in $(t, x)$, and compactly supported in $B(0, R+1)$. Therefore, the function $w^{g_{R}}$ defined using $\left(f_{R}, \ell_{R}, g_{R}\right)$ satisfies $w^{g_{R}}(t, x, z) \leq 0 \Leftrightarrow w^{g}(t, x, z) \leq 0$, and it can be used for the characterization of $\vartheta(t, x)$.

Let us point out that the value function $w^{g}$ depends on the choice of $g$. However, the set

$$
\left\{(x, z), w^{g}(t, x, z) \leq 0\right\}
$$

is independent of the choice of $g$.

Lemma 3.7. If $\tilde{g}$ and $g$ are Lipschitz continuous function satisfying (3.1), then for all $t \leq T$,

$$
\left\{(x, z), w^{g}(t, x, z) \leq 0\right\}=\left\{(x, z), w^{\tilde{g}}(t, x, z) \leq 0\right\} .
$$

Remark 3.8 (Extension to terminal state constraints). Consider the case when the state variable is constrained to satisfy a terminal constraint $y(T) \in \mathcal{C}$, in addition to $(2.2)$, where $\mathcal{C}$ is a closed subset of $\mathbb{R}^{d}$ (of course $\mathcal{C} \cap \mathcal{K}$ has to be considered nonempty, otherwise the problem becomes trivial). Then the control problem is defined by:

$$
\vartheta(t, x):=\inf \left\{\int_{t}^{T} \ell\left(s, y_{t, x}^{\alpha}(s), \alpha(s)\right) \mathrm{d} s+\varphi\left(y_{t, x}^{\alpha}(T)\right) \mid \alpha \in \mathbb{A}, y_{t, x}^{\alpha}(T) \in \mathcal{C} \text { and } y_{t, x}^{\alpha}(s) \in \mathcal{K}, \forall s \in[t, T]\right\} .
$$


In this case, one should consider a Lipschitz continuous function $\psi: \mathbb{R}^{d} \rightarrow \mathbb{R}$ such that $\psi(x) \leq 0 \Longleftrightarrow x \in \mathcal{C}$ (since $\mathcal{C}$ is closed, such a Lipschitz function $\psi$ exists). Hence the auxiliary control problem can be considered as:

$$
w^{g}(t, x, z):=\inf _{\hat{y}=(y, \zeta) \in \hat{S}_{[t, T]}(x, z)}(\varphi(y(T))-\zeta(T)) \bigvee \max _{\theta \in(t, T)} g(y(\theta)) \bigvee \psi(y(T))
$$

Here again, the description of the value function $\vartheta$ is given by:

$$
\mathcal{E} p i(\vartheta(t, \cdot))=\left\{(x, z) \in \mathbb{R}^{d} \times \mathbb{R} \mid w^{g}(t, x, z) \leq 0\right\},
$$

and $w^{g}$ satisfies the same HJB equation as in Proposition 3.4 excepted the fact that the final condition at time $t=T$ is now: $w^{g}(T, x, z)=(\varphi(x)-z) \vee g(x) \vee \psi(x)$.

Before concluding this section, we would like to stress on the fact that considering the auxiliary function $w^{g}$ allows to bypass all the regularity issues which arise when the control problem is in presence of state constraints. This constructive way to describe the value function $\vartheta$ involves only the classical framework of Lipschitz continuous viscosity solution, even though $\vartheta$ is only l.s.c. This approach opens the way for computing the epigraph of $\vartheta$ by a large panel of schemes for continuous viscosity solutions. One can criticize the fact that the definition of $w^{g}$ involves one more state component, however it is important to keep in mind that only the level set $\left\{w^{g}(t, x, z)=0\right\}$ is needed. Since $w^{g}$ is Lipschitz continuous, one can use a local numerical scheme (as in $[14,37])$ to track this 0 -level set in $\mathbb{R}^{d+1}$ and reduce the numerical complexity to almost the same numerical complexity of solving an HJ equation in a $d$-dimensional space.

\section{INFINITE HORIZON PROBLEM WITH STATE CONSTRAINTS}

Here we consider the case of an infinite horizon optimal control problem with state constraints (see [6] for standard results) and assume throughout this section that $f, \ell$ satisfies (A1)-(A2) and (A4), and that these functions do not depend on the time variable:

$$
f: \mathbb{R}^{d} \times \mathcal{A} \rightarrow \mathbb{R}^{d}, \quad \ell: \mathbb{R}^{d} \times \mathcal{A} \rightarrow \mathbb{R} .
$$

Let $\mathbb{A}$ be the set of measurable controls $\alpha:(0, \infty) \rightarrow \mathcal{A}$. For any $\alpha \in \mathbb{A}$, consider the system obeying:

$$
\begin{aligned}
& \dot{y}(s)=f(y(s), \alpha(s)) \quad \text { a.e. } s \in(0, \infty), \\
& y(0)=x,
\end{aligned}
$$

and let $y=y_{x}^{\alpha}$ denotes its solution. Let also $\mathcal{K}$ be a nonempty closed set of $\mathbb{R}^{d}$. Set $L$ is assumed to be a majorant of the Lipschitz constants for $f$ and $\ell$ with respect to the $x$ variable, as stated in (A1)-(A2), and set $\lambda>L$. The infinite horizon control problem is:

$$
\widetilde{\vartheta}(x):=\min _{\alpha \in \mathbb{A}}\left\{\int_{0}^{\infty} \mathrm{e}^{-\lambda s} \ell\left(y_{x}^{\alpha}(s), \alpha(s)\right) \mathrm{d} s \mid y_{x}^{\alpha}(\theta) \in \mathcal{K} \quad \forall \theta \in(0, \infty)\right\} .
$$

(the existence of a minimum when $\tilde{v}<\infty$ can be obtained by using the compact injection of $S_{[0, \infty)}(x)$ into $C\left(\left[0, \infty[)\right.\right.$ for the $W^{1,1}$ weighted norm $\|y\|_{W^{1,1}}=\int_{0}^{\infty}(|y(s)|+|\dot{y}(s)|) \mathrm{e}^{-\lambda s} \mathrm{~d} s$, see for instance [4]).

We again focus on the characterization of the epigraph of $\widetilde{\vartheta}$ :

$$
\mathcal{E} \operatorname{pi}(\widetilde{\vartheta}):=\left\{(x, z) \in \mathbb{R}^{d} \times \mathbb{R}, \widetilde{\vartheta}(x) \leq z\right\} .
$$

Let $g: \mathbb{R}^{d} \rightarrow \mathbb{R}$ be a Lipschitz continuous function satisfying (3.1). We introduce an auxiliary control problem with value function $\widetilde{w}^{g}$ defined for $x \in \mathbb{R}^{d}, z \in \mathbb{R}$, and $\lambda>L$, by:

$$
\widetilde{w}^{g}(x, z):=\min _{\alpha \in \mathbb{A}}\left(\int_{0}^{\infty} \mathrm{e}^{-\lambda s} \ell\left(y_{x}^{\alpha}(s), \alpha(s)\right) \mathrm{d} s-z\right) \bigvee \sup _{\theta \in(0, \infty)}\left(\mathrm{e}^{-\lambda \theta} g\left(y_{x}^{\alpha}(\theta)\right)\right) .
$$


Theorem 4.1. Assume that (A1)-(A2) and (A4) hold, $\mathcal{K}$ is closed and non-empty, and $\lambda>L$.

(i) For any $(x, z) \in \mathbb{R}^{d} \times \mathbb{R}$, the following holds:

$$
\widetilde{\vartheta}(x)-z \leq 0 \quad \Longleftrightarrow \quad \widetilde{w}^{g}(x, z) \leq 0 .
$$

Moreover, the function $\widetilde{\vartheta}$ is characterized by $\widetilde{w}^{g}$ through the following relation

$$
\widetilde{\vartheta}(x)=\min \left\{z \in \mathbb{R}, \widetilde{w}^{g}(x, z) \leq 0\right\}
$$

(ii) $\widetilde{w}^{g}$ is Lipschitz continuous on $\mathbb{R}^{d} \times \mathbb{R}$;

(iii) the function $u=\widetilde{w}^{g}$ is the unique Lipschitz continuous viscosity solution of

$$
\min \left(\lambda u+\max _{a \in \mathcal{A}}\left(-f(x, a) \cdot \nabla_{x} u-(\lambda z-\ell(x, a)) \partial_{z} u\right), \quad u(x, z)-g(x)\right)=0, \quad x \in \mathbb{R}^{d}, z \in \mathbb{R} .
$$

The derivation of the HJ equation for $\widetilde{w}^{g}$ is based on the following DPP principle. We define $\zeta_{x, z}^{\alpha}(t):=$ $\mathrm{e}^{\lambda t} z-\int_{0}^{t} \mathrm{e}^{\lambda(t-s)} \ell\left(y_{x}^{\alpha}(s), \alpha(s)\right) \mathrm{d} s$. Equivalently, $\zeta(t)=\zeta_{x, z}^{\alpha}(t)$ is the absolutely continuous solution of

$$
\begin{aligned}
& \dot{\zeta}(s)=\lambda \zeta(s)-\ell\left(y_{x}^{\alpha}(s), \alpha(s)\right) \quad \text { a.e. } s>0 \\
& \zeta(0)=z .
\end{aligned}
$$

Lemma 4.2 (Dynamic programming principle). For all $h \geq 0$ and $(x, z) \in \mathbb{R}^{d} \times \mathbb{R}$, we have

$$
\begin{aligned}
\widetilde{w}^{g}(x, z)= & \min \left\{\left(\mathrm{e}^{-\lambda h} \widetilde{w}^{g}\left(y_{x}^{\alpha}(h), \zeta_{(x, z)}^{\alpha}(h)\right)\right) \bigvee \max _{\theta \in(0, h)}\left(\mathrm{e}^{-\lambda \theta} g\left(y_{x}^{\alpha}(\theta)\right)\right),\right. \\
& \left.\alpha:(0, h) \rightarrow \mathcal{A} \text { measurable, }\left(y_{x}^{\alpha}, \zeta_{(x, z)}^{\alpha}\right) \text { solution of }(4.5)\right\} .
\end{aligned}
$$

Proof. The definition of $\widetilde{w}^{g}$ involves the maximum between two terms, and the DPP presented here seems to be new. Even though the proof is not difficult we prefer to present it for sake of completeness.

For any measurable control $\alpha:(0, \infty) \rightarrow \mathcal{A}$, we shall denote $\alpha_{1}$ the restriction of $\alpha$ on $(0, h)$ and $\alpha_{2}$ the measurable control of $\mathcal{A}$ such that $\alpha_{2}(t)=\alpha(t+h)$ a.e. $t \geq 0$. Using $y_{x}^{\alpha}(s+h)=y_{y_{x}^{\alpha}(h)}^{\alpha_{2}}(s)$, we obtain on the first hand:

$$
\begin{aligned}
\int_{0}^{\infty} \mathrm{e}^{-\lambda s} \ell\left(y_{x}^{\alpha}(s), \alpha(s)\right) \mathrm{d} s-z & =\int_{h}^{\infty} \mathrm{e}^{-\lambda s} \ell\left(y_{x}^{\alpha}(s), \alpha(s)\right) \mathrm{d} s+\int_{0}^{h} \mathrm{e}^{-\lambda s} \ell\left(y_{x}^{\alpha}(s), \alpha(s)\right) \mathrm{d} s-z \\
& =\mathrm{e}^{-\lambda h}\left(\int_{0}^{\infty} \mathrm{e}^{-\lambda s} \ell\left(y_{x}^{\alpha}(s+h), \alpha(s+h)\right) \mathrm{d} s-\zeta_{x, z}^{\alpha_{1}}(h)\right) \\
& =\mathrm{e}^{-\lambda h}\left(\int_{0}^{\infty} \mathrm{e}^{-\lambda s} \ell\left(y_{y_{x}^{\alpha_{1}}}^{\alpha_{2}}(s), \alpha_{2}(s)\right) \mathrm{d} s-\zeta_{x, z}^{\alpha_{1}}(h)\right)
\end{aligned}
$$

and on the other hand:

$$
\begin{aligned}
\sup _{\theta \in(0, \infty)} \mathrm{e}^{-\lambda \theta} g\left(y_{x}^{\alpha}(\theta)\right) & =\sup _{\theta \in(0, \infty)} \mathrm{e}^{-\lambda(\theta+h)} g\left(y_{x}^{\alpha}(\theta+h)\right) \bigvee \max _{\theta \in(0, h)} \mathrm{e}^{-\lambda \theta} g\left(y_{x}^{\alpha_{1}}(\theta)\right) \\
& =\left(\mathrm{e}^{-\lambda h} \sup _{\theta \in(0, \infty)} \mathrm{e}^{-\lambda \theta} g\left(y_{y_{x}^{\alpha_{1}}(h)}^{\alpha_{2}}(\theta)\right)\right) \bigvee \max _{\theta \in(0, h)} \mathrm{e}^{-\lambda \theta} g\left(y_{x}^{\alpha_{1}}(\theta)\right) .
\end{aligned}
$$


Combining (4.7) and (4.8), taking measurable controls $\alpha_{1}:(0, h) \rightarrow \mathcal{A}$ and $\alpha_{2} \in \mathbb{A}$, we obtain

$$
\begin{aligned}
\widetilde{w}^{g}(x, z)= & \inf _{\alpha_{1}} \inf _{\alpha_{2}}\left(\mathrm{e}^{-\lambda h} \int_{0}^{\infty} \mathrm{e}^{-\lambda s} \ell\left(y_{y_{x}^{\alpha_{1}}}^{\alpha_{2}}(s), \alpha_{2}(s)\right) \mathrm{d} s-\zeta_{x, z}^{\alpha_{1}}(h)\right) \bigvee\left(\mathrm{e}^{-\lambda h} \sup _{\theta \in(0, \infty)} \mathrm{e}^{-\lambda \theta} g\left(y_{y_{x}^{\alpha_{1}}(h)}^{\alpha_{2}}(\theta)\right)\right) \\
& \bigvee_{\theta \in(0, h)} \max ^{-\lambda \theta} g\left(y_{x}^{\alpha_{1}}(\theta)\right) \\
= & \inf _{\alpha_{1}}\left(\mathrm{e}^{-\lambda h} \widetilde{w}^{g}\left(y_{x}^{\alpha_{1}}(h), \zeta_{x, z}^{\alpha_{1}}(h)\right)\right) \bigvee \max _{\theta \in(0, h)} \mathrm{e}^{-\lambda \theta} g\left(y_{x}^{\alpha_{1}}(\theta)\right)
\end{aligned}
$$

which is the desired result.

Remark 4.3 (viability and link with optimal stopping time). In the particular case when $\ell \equiv 0$, the value function (4.2) characterizes the viability kernel of the dynamics $f$ in the set $\mathcal{K}$ :

$$
\operatorname{Viab}_{\mathcal{K}}^{f}:=\left\{x \in \mathbb{R}^{d}, \exists y \in S_{[0, \infty[}(x), y(t) \in \mathcal{K} \forall t \in(0, \infty)\right\} .
$$

In this case, and as in Section 3.2, the penalized problem can be defined in the following form:

$$
\tilde{u}(x):=\min _{\alpha \in \mathbb{A}} \sup _{\theta \in(0, \infty)} \mathrm{e}^{-\lambda \theta} g\left(y_{x}^{\alpha}(\theta)\right)
$$

without adding a new state component. One can show that $\tilde{u}$ is the unique viscosity solution of

$$
\min \left(\lambda \tilde{u}+\max _{a \in \mathcal{A}}\left(-f(x, a) \cdot \nabla_{x} \tilde{u}\right), \quad \tilde{u}(x)-g(x)\right)=0 \quad x \in \mathbb{R}^{d},
$$

and the viability kernel satisfies $\operatorname{Viab}_{\mathcal{K}}^{f}=\{x, \tilde{u}(x) \leq 0\}$.

In this particular case, the problem (4.9) can be also interpreted as a stopping-time problem [6], Chap. III, Section 4.2, see also Remark 3.5.

\section{Two-Player games With State COnstraints}

The approach presented in the previous sections can be extended to more general control problems lacking controllability assumptions. We consider here the case of two-player games $[6,7,21,25,41,42]$.

In addition to the player taking control values in $\mathcal{A}$, we consider $\mathcal{B}$ a non empty compact set of $\mathbb{R}^{m}(m \geq 1)$, and the set controls for the second player:

$$
\mathbb{B}:=\left\{\beta:(0, T) \rightarrow \mathbb{R}^{m} \text { measurable, } \beta(t) \in \mathcal{B} \text { a.e. }\right\}
$$

We also consider a new dynamics $f$, distributed cost $\ell$, and terminal cost $\varphi$ such that:

$$
\begin{aligned}
& \left(A_{1}^{\prime}\right)\left\{\begin{array}{c}
(i)(t, x, a, b) \in[0, T] \times \mathbb{R}^{d} \times \mathcal{A} \times \mathcal{B} \rightarrow f(t, x, a, b) \in \mathbb{R}^{d} \text { is continuous } \\
(i i) \exists L \geq 0, \forall x, y \in \mathbb{R}^{d}, \forall(a, b) \in \mathcal{A} \times \mathcal{B}, \forall t, s \in[0, T], \\
|f(t, x, a, b)-f(s, y, a, b)| \leq L(|x-y|+|t-s|),
\end{array}\right. \\
& \left(A_{2}^{\prime}\right)\left\{\begin{array}{c}
(i)(t, x, a, b) \in[0, T] \times \mathbb{R}^{d} \times \mathcal{A} \times \mathcal{B} \rightarrow \ell(t, x, a, b) \in \mathbb{R}^{d} \text { is continuous } \\
(i i) \exists L \geq 0, \forall x, y \in \mathbb{R}^{d}, \forall(a, b) \in \mathcal{A} \times \mathcal{B}, \forall t, s \in[0, T], \\
|\ell(t, x, a, b)-\ell(s, y, a, b)| \leq L(|x-y|+|t-s|),
\end{array}\right. \\
& \left(A_{3}^{\prime}\right) \varphi: \mathbb{R}^{d} \rightarrow \mathbb{R} \text { is Lipschitz continuous. }
\end{aligned}
$$

Again, we consider the "augmented" dynamics $\hat{f}:[0, T] \times \mathbb{R}^{d+1} \times \mathcal{A} \times \mathcal{B} \rightarrow \mathbb{R}^{d+1}$ defined as follows:

$$
\hat{f}(t,(x, z), a, b):=\left(\begin{array}{c}
f(t, x, a, b) \\
-\ell(t, x, a, b)
\end{array}\right), \quad \forall(x, z) \in \mathbb{R}^{d} \times \mathbb{R}, a \in \mathcal{A}, b \in \mathcal{B}, t \in(0, T) .
$$


The following convexity assumption of $\hat{f}$ will be considered:

$$
\left(A_{4}^{\prime}\right) \forall t, x, \beta, \quad \hat{f}(t, x, \mathcal{A}, \beta) \text { is a convex set. }
$$

Notice that this includes the case when $f(t, x, a, b)=f_{1}(t, x, b) \cdot a+f_{2}(t, x, b)$ and $\mathcal{A}$ is a convex set.

For every $x \in \mathbb{R}^{d}$ and $(\alpha, \beta) \in \mathbb{A}_{t} \times \mathbb{B}$ we define the trajectory $y=y_{t, x}^{\alpha, \beta}$ as the solution of

$$
\dot{y}(s)=f(s, y(s), \alpha(s), \beta(s)) \text { for a.e. } s \in[t, T], \quad y(t)=x,
$$

in the Carathéodory sense.

Following the formulation of Elliott and Kalton [25], we define the set of non-anticipative strategies $a \equiv a[\cdot]$ as follows:

$$
\begin{aligned}
\Gamma_{t} & :=\{a: \mathbb{B} \rightarrow \mathbb{A}, \forall(\beta, \tilde{\beta}) \in \mathbb{B}, \text { and } \forall s \in[t, T],(\beta(\theta)=\tilde{\beta}(\theta) \text {. a.e. } \theta \in[t, s]) \\
& \Rightarrow(a[\beta](\theta)=a[\tilde{\beta}](\theta), \text { a.e. on }[t, s])\} .
\end{aligned}
$$

The value function for the first player is given by:

$$
\bar{\vartheta}(t, x):=\inf _{a \in \Gamma_{t}} \max _{\beta \in \mathbb{B}}\left\{\int_{t}^{T} \ell\left(s, y_{t, x}^{a[\beta], \beta}(s), \alpha(s), \beta(s)\right) \mathrm{d} s+\varphi\left(y_{t, x}^{a[\beta], \beta}(T)\right) \mid y_{t, x}^{\alpha}(s) \in \mathcal{K}, \forall s \in[t, T]\right\} .
$$

As in Section 3, we add a new state variable $z \in \mathbb{R}$, and we define the following auxiliary control problem:

$$
\bar{w}^{g}(t, x, z):=\inf _{a \in \Gamma_{t}} \max _{\beta \in \mathbb{B}}\left(\varphi\left(y_{t, x}^{a[\beta], \beta}(T)\right)-\zeta_{t,(x, z)}^{a[\beta], \beta}(T)\right) \bigvee \max _{\theta \in[t, T]} g\left(y_{t, x}^{a[\beta], \beta}(\theta)\right),
$$

where $\hat{f}=(f,-\ell)^{\top}$ and $\hat{y}=\hat{y}_{t,(x, z)}^{\alpha, \beta} \equiv\left(y_{t, x}^{\alpha, \beta}, \zeta_{t,(x, z)}^{\alpha, \beta}\right)^{\top}$ is an absolutely continuous solution of

$$
\begin{aligned}
& \dot{\hat{y}}(s)=\hat{f}(s, \hat{y}(s), \alpha(s), \beta(s)) \quad \text { a.e. } s \in(t, T), \\
& \hat{y}(t)=(x, z)^{\top} .
\end{aligned}
$$

In a similar way to the case of a one-player game, one can check easily that we have:

Lemma 5.1. Let $\left(A_{1}^{\prime}\right)-\left(\bar{A}_{4}^{\prime}\right)$ hold.

(i) $\bar{\vartheta}$ is a lower semi-continuous function;

(ii) the infimum in (5.3) is reached. In particular,

$$
\begin{gathered}
\bar{w}^{g}(t, x, z) \leq 0 \Longleftrightarrow \exists a[\cdot] \in \Gamma_{t}, \forall \beta \in \mathbb{B}, \int_{t}^{T} \ell\left(s, y_{t, x}^{a[\beta], \beta}(s), \alpha(s), \beta(s)\right) \mathrm{d} s+\varphi\left(y_{t, x}^{a[\beta], \beta}(T)\right) \leq z, \\
y_{t, x}^{a[\beta], \beta}(\theta) \in \mathcal{K}, \forall \theta \in[t, T] .
\end{gathered}
$$

Therefore the value $\bar{w}^{g}(t, x, z)$ is reached by some non-anticipative strategy, as soon as $\varphi$ and $g$ are continuous. By using arguments similar to those of the previous sections, we get:

Theorem 5.2. Assume $\left(A_{1}^{\prime}\right)-\left(A_{4}^{\prime}\right)$, and g Lipschitz continuous.

(i) $\forall t \in[0, T], \forall(x, z) \in \mathbb{R}^{d} \times \mathbb{R}$,

$$
\bar{\vartheta}(t, x) \leq z \quad \Leftrightarrow \quad \bar{w}^{g}(t, x, z) \leq 0 ;
$$


(ii) the function $\bar{w}^{g}$ is locally Lipschitz continuous on $[0, T] \times \mathbb{R}^{d} \times \mathbb{R}$, more precisely there exists $C \geq 0$ such that

$$
\left|\bar{w}^{g}\left(t^{\prime}, \hat{x}^{\prime}\right)-\bar{w}^{g}(t, \hat{x})\right| \leq C(1+|\hat{x}|)\left(\left|t^{\prime}-t\right|+\left|\hat{x}^{\prime}-\hat{x}\right|\right) ;
$$

In particular, $\bar{w}^{g}$ has a linear growth $:\left|\bar{w}^{g}(t, \hat{x})\right| \leq C(1+|\hat{x}|)$ for some constant $C \geq 0$.

(iii) Furthermore, $u=\bar{w}^{g}$ is the unique continuous viscosity solution of the following HJ equation:

$$
\begin{aligned}
& \min \left(-\partial_{t} u+\overline{\mathcal{H}}(t,(x, z), \nabla u), u-g(x)\right)=0, \quad t \in(0, T), x \in \mathbb{R}^{d}, z \in \mathbb{R}, \\
& u(T, x, z)=\max (\varphi(x)-z, g(x)), \quad x \in \mathbb{R}^{d} .
\end{aligned}
$$

where $\overline{\mathcal{H}}(t,(x, z),(p, q)):=\max _{\alpha \in \mathcal{A}} \min _{\beta \in \mathcal{B}}-\hat{f}(t, x, \alpha, \beta) \cdot(p, q) \equiv \max _{\alpha \in \mathcal{A}} \min _{\beta \in \mathcal{B}}-f(t, x, \alpha, \beta) \cdot p+\ell(t, x, \alpha, \beta) q$.

Corollary 5.3. Under $\left(A_{1}^{\prime}\right)-\left(A_{4}^{\prime}\right)$, the function $\bar{\vartheta}$ is characterized by means of the function $\bar{w}^{g}$ through the following relation

$$
\bar{\vartheta}(t, x)=\inf \left\{z \in \mathbb{R}, \bar{w}^{g}(t, x, z) \leq 0\right\}
$$

This gives again a characterization of $\bar{\vartheta}$ for two-player games with state constraints, by using a continuous viscosity approach and without any controllability assumption.

\section{Numerical EXAMPLe}

We consider the classical Zermelo type problem. A boat with coordinates $y(t)=\left(y_{1}(t), y_{2}(t)\right)$ navigates in a canal $\mathbb{R} \times[-2,2]$, starting from $y(0)=x=\left(x_{1}, x_{2}\right)$, and wants to reach an island $B=\bar{B}\left(0, r_{0}\right), r_{0}>0$, with minimal fuel consumption. The dynamics is given by

$$
\begin{aligned}
& \dot{y}_{1}=v \cos (u)+c-a y_{2}^{2}, \\
& \dot{y}_{2}=v \sin (u),
\end{aligned}
$$

where $u \in[0,2 \pi]$ is the first control (angle), $v \in\left[0, V_{\max }\right]$ is a second control (the speed of the boat), and $c-a y_{2}^{2}$ is the current drift (along the $x_{1}$-axis). We shall choose the parameters $V_{\max }=1$ and $c=2, a=0.5$. The boundary $y_{2}= \pm \sqrt{\frac{c}{a}} \equiv \pm 2$ (where the drift term $c-a y_{1}^{2}$ vanishes) corresponds to the coast. The evolution of the fuel mass $m(t)$ is given by

$$
\dot{m}=-b \frac{v}{V_{\max }},
$$

where $b=1$ and $\frac{v}{V_{\max }}$ is a speed ratio. Therefore, the fuel consumption $\int_{t}^{T}|\dot{m}(s)| \mathrm{d} s$ is proportional to

$$
\int_{t}^{T} v(s) \mathrm{d} s
$$

Because of the drift term (which can be greater than $V_{\max }$ ), the system is not controllable. Consider the set of constraints given by $\mathcal{K}:=\left\{x \in \mathbb{R}^{2}, g(x) \leq 0\right\}$ where

$$
g(x):=\max \left(r_{a}-\|x-a\|_{\infty}\right), r_{b}-\max \left(\left|x_{1}-b_{1}\right|, \frac{1}{5}\left|x_{2}-b_{2}\right|\right)
$$

and where $r_{a}=0.4, a=(-0.5,0.5)$ and $r_{b}=0.2, b=(-1,-1.5)$. The target $\mathcal{C} \equiv B\left(c, r_{0}\right)$ with $r_{0}=0.25$ and $c=(1.5,0)$ is represented by a function $\psi$ defined by

$$
\psi(x):=\|x-c\|-r_{0} .
$$



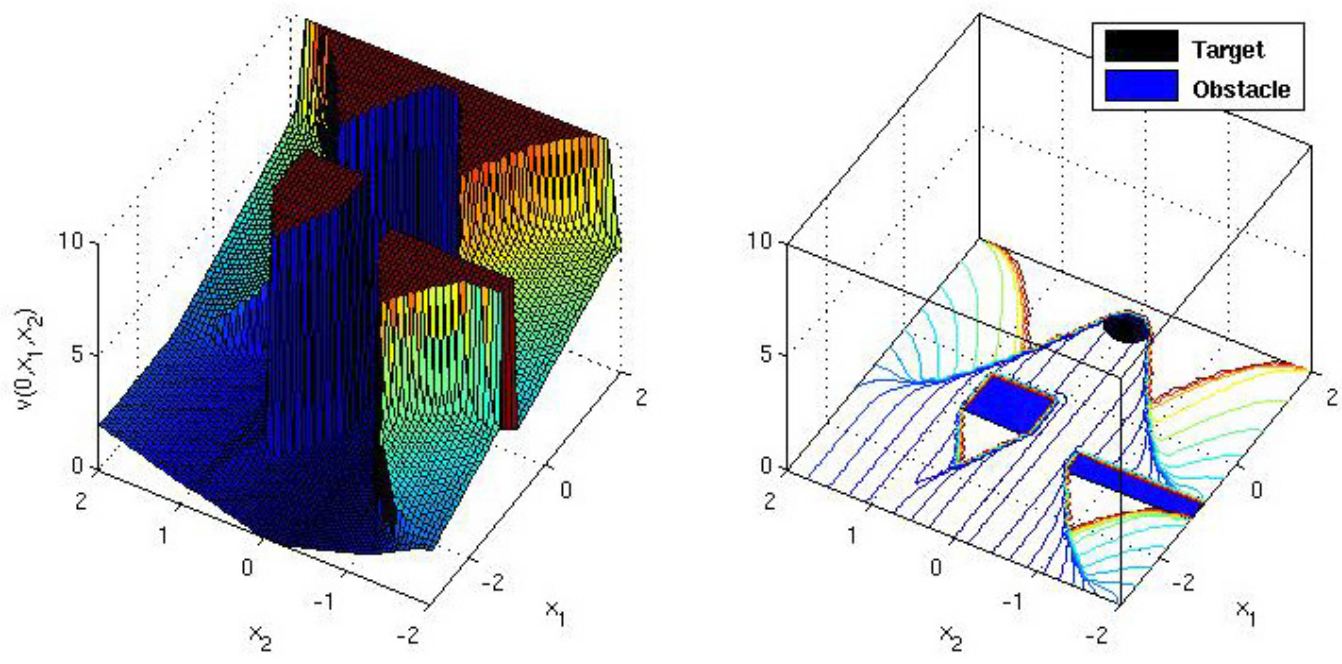

Figure 1. Left: values of $v(t,$.$) at time t=0$. Right: iso-values in logarithmic scale. Here the values such that $\vartheta\left(0, x_{1}, x_{2}\right)=10$ correspond to a non-reachable zone.

The optimal control problem is to minimize, whenever this is possible, the following

$$
\vartheta(t, x)=\inf \left\{\int_{t}^{T} v(s) \mathrm{d} s, \alpha=(u, v) \in \mathbb{A}, y_{x}^{\alpha}(T) \in \mathcal{C},\left(y_{x}^{\alpha}(\theta) \in \mathcal{K}, \forall \theta \in[t, T]\right)\right\}
$$

where $\mathbb{A}$ is the set of measurable controls $\alpha=(u, v):(0, T) \rightarrow[0,2 \pi] \times[0,1]$. Therefore we are in the situation of Section 3.8. We consider $\ell(t, x,(u, v)):=v$, a terminal cost $\varphi(x) \equiv 0$, and the PDE becomes

$$
\begin{gathered}
\min \left(-w_{t}+\max \left(0,\left\|\nabla_{x} w\right\|+w_{z}\right)-\left(c-a y_{2}^{2}\right) w_{y_{1}}, w(t, x, z)-g(x)\right)=0, \\
t \in(0, T),(x, z) \in \mathbb{R}^{2} \times \mathbb{R}, \\
w(T, x, z)=\max (-z, \psi(x), g(x)), \quad(x, z) \in \mathbb{R}^{2} \times \mathbb{R} .
\end{gathered}
$$

This last HJ equation is then solved by a finite difference method (ENO scheme of second order in space, see for instance [15]). Results are shown in Figure 1, at time $t=0$ with $T=10$ (computations done with $70^{3}$ grid point). The value function is recovered finally using $\vartheta(t, x)=\min \{z \in \mathbb{R}, w(t, x, z) \leq 0\}$.

We have considered a discretisation of $70^{3}$ spatial mesh points of the domain $[-3,2] \times[-2,2] \times\left[Z_{\min }, Z_{\max }\right]$ where $Z_{\min }=-0.1$ and $Z_{\max }=10$. The time interval is $[0, T] \equiv[0,10]$. This example was solved by using the $\mathrm{C}++$ HJB-solver "Binope-HJ" [17].

Acknowledgements. This work was partially supported by the EU under the 7th Framework Programme Marie Curie Initial Training Network "FP7-PEOPLE-2010-ITN", SADCO project, GA number 264735-SADCO.

\section{Appendix A. COMPARISOn PRINCIPle For HJ EQUations With obstacle terms}

The aim of this section is to prove a comparison principle for the following HJ equation in presence of an obstacle term:

$$
\begin{aligned}
& \min \left(-u_{t}+H(t, x, \nabla u), u-g(t, x)\right)=0 \quad \text { on }(0, T) \times \mathbb{R}^{d}, \\
& u(T, x)=u_{0}(x), \quad x \in \mathbb{R}^{d}
\end{aligned}
$$


where $T>0$ and $g \in C\left((0, T) \times \mathbb{R}^{d}\right)$, and $H:(0, T) \times \mathbb{R}^{d} \times \mathbb{R}^{d} \rightarrow \mathbb{R}$ is continuous and assumed to satisfy:

(H1) there exists $C \geq 0$ such that, for all $(t, x)$ in $(0, T) \times \mathbb{R}^{d}, p, q \in \mathbb{R}^{d}$,

$$
|H(t, x, p)-H(t, x, q)| \leq C(|x|+1)|p-q|
$$

$(\mathbf{H 2})$ for any $R>0$, there exists a function $w_{R}:[0, \infty) \rightarrow[0, \infty), \lim _{r \rightarrow 0+} w_{R}(r)=0$ and

$$
|H(t, x, p)-H(t, y, p)| \leq w_{R}((1+|p|)|x-y|)
$$

for every $(t, p) \in(0, T) \times \mathbb{R}^{d}, x, y \in B_{R}$, where $B_{R}$ denotes the open ball centered at 0 and of radius $R$.

Theorem A.1. Let $u, v$ be two functions of $C\left([0, T] \times \mathbb{R}^{d}\right)$, and let $g, h$ be in $C\left([0, T] \times \mathbb{R}^{d}\right)$. We assume that $u$ (resp. $v$ ) is a sub-solution (resp. super-solution) of (A.1) in $(0, T) \times \mathbb{R}^{d}$ :

$$
\begin{array}{cl}
\min \left(-u_{t}+H(t, x, \nabla u), u-g\right) \leq 0 \quad \text { in }(0, T) \times \mathbb{R}^{d}, \\
\min \left(-v_{t}+H(t, x, \nabla v), v-h\right) \geq 0 \quad \text { in }(0, T) \times \mathbb{R}^{d} .
\end{array}
$$

We denote $u_{T}(x):=u(T, x)$ and $v_{T}(x):=v(T, x)$. Then for all $t \in[0, T]$,

$$
\sup _{\mathbb{R}^{d}}(u(t, .)-v(t, .)) \leq \max \left(\sup _{\mathbb{R}^{d}}\left(u_{T}-v_{T}\right), \sup _{(t, T) \times \mathbb{R}^{d}}(g-h)\right) .
$$

Proof. The result without the obstacle term can be found for instance in Ishii [30]. It suffices to prove the result for $T>0$ small enough, the result for any $T>0$ can then be deduced by immediate recursion.

Assuming that $C>0$, we take $T=1 /(2 C)$ and $L=L\left(x_{0}\right):=2 C\left(\left|x_{0}\right|+1\right)$, and we define the following cone

$$
\mathcal{O}_{x_{0}}:=\left\{(t, x) \in(0, T) \times \mathbb{R}^{d},\left|x-x_{0}\right|<L t\right\} .
$$

(The case when $C=0$ is trivial).

We claim that for every $t_{0} \in(0, T)$ :

$$
u\left(t_{0}, x_{0}\right)-v\left(t_{0}, x_{0}\right) \leq \max \left(\sup _{x_{0}+B_{L\left(T-t_{0}\right)}}\left(u_{T}-v_{T}\right), \sup _{\mathcal{O}_{x_{0}}}(g-h)\right),
$$

which concludes (A.7). Let us consider $t_{0} \in(0, T)$ and prove our claim.

First, remark that for any $(t, x) \in \mathcal{O}_{x_{0}}$, the following holds: $C(|x|+1) \leq C\left(\left|x_{0}\right|+1\right)+C\left|x-x_{0}\right| \leq \frac{L}{2}+C L T \leq$ $\frac{L}{2}+\frac{1}{2} L=L$, and thus

$$
|H(t, x, p)-H(t, x, q)| \leq L|p-q| \quad(t, x) \in \mathcal{O}_{x_{0}} .
$$

We also define for any $(\bar{t}, \bar{x}) \in \Omega_{x_{0}}$ and $\tau \in(\bar{t}, T)$ the following cone:

$$
\mathcal{O}_{\bar{t}, \bar{x}, \tau}:=\left\{(t, x) \in(\bar{t}, \tau) \times \mathbb{R}^{d},\left|x-x_{0}\right|<L(t-\bar{t})\right\} .
$$

According to Crandall and Lions [23], and Ishii [30], the following Lemma holds.

Lemma A.2. If $u, v$ belongs to $C\left(\overline{\mathcal{O}}_{\bar{t}, \bar{x}, t}\right)$ with $t \in(\bar{t}, T)$, and are respectively viscosity solutions of

$$
\begin{array}{ll}
-u_{t}+H(t, x, \nabla u) \leq 0 & \text { in } \mathcal{O}_{\bar{t}, \bar{x}, t}, \\
-v_{t}+H(t, x, \nabla v) \geq 0 & \text { in } \mathcal{O}_{\bar{t}, \bar{x}, t}
\end{array}
$$

then

$$
u(\bar{t}, \bar{x})-v(\bar{t}, \bar{x}) \leq \sup _{\bar{x}+B_{L(t-\bar{t})}}(u(t, .)-v(t, .)) .
$$


Consider the set:

$$
\Sigma:=\left\{(t, x) \in \overline{\mathcal{O}}_{x_{0}}, u(t, x) \leq g(x)\right\}
$$

and its complementary in $\mathcal{O}_{x_{0}}$ :

$$
\Omega:=\mathcal{O}_{x_{0}} \backslash \Sigma \text {. }
$$

Since $u$ is a sub-solution of (A.5), $u$ is also a sub-solution of $u_{t}+H(t, x, \nabla u)=0$ on the open set $\Omega$. Furthermore $v$ being a super-solution of (A.6) on $\mathcal{O}_{x_{0}}$, it is also a super-solution of $v_{t}+H(t, x, \nabla v)=0$ on the open set $\Omega$.

On the other hand, from (A.6), it follows that $v(t, x) \geq g(x)$ everywhere. Hence

$$
\forall(t, x) \in \Sigma, \quad u(t, x)-v(t, x) \leq g(t, x)-h(t, x) \leq \sup _{\mathcal{O}_{x_{0}}}(g-h) .
$$

Now, assume that

$$
u\left(t_{0}, x_{0}\right)-v\left(t_{0}, x_{0}\right)>M:=\max \left(\sup _{x_{0}+B_{L\left(T-t_{0}\right)}}\left(u_{0}-v_{0}\right), \sup _{\mathcal{O}_{x_{0}}}(g-h)\right),
$$

and define, for $\tau \in[0, T]$ (with $\tau>t_{0}$ ), the following cone:

$$
\mathcal{O}_{t_{0}, x_{0}, \tau}:=\left\{(t, x) \in\left(t_{0}, \tau\right) \times \mathbb{R}^{d},\left|x-x_{0}\right|<L\left(t-t_{0}\right)\right\} .
$$

Using the continuity of $u-v$ in $\left(t_{0}, x_{0}\right)$, there exists a neighborhood $\Upsilon$ of $\left(t_{0}, x_{0}\right)$ in $\mathcal{O}_{x_{0}}$ satisfying:

$$
u(t, x)-v(t, x)>M \geq \sup _{\mathcal{O}_{x_{0}}}(g-h) \quad \forall(x, t) \in \Upsilon .
$$

Taking into account (A.12), it follows that $\Upsilon$ is necessarily included in $\Omega$. Hence there exists $\tau>t_{0}$ such that the cone $\mathcal{O}_{t_{0}, x_{0}, \tau}$ is also included in $\Omega$. Set

$$
t_{1}:=\sup \left\{\tau \in\left(t_{0}, T\right], \mathcal{O}_{t_{0}, x_{0}, \tau} \bigcap \Gamma=\emptyset\right\} .
$$

$\left(\mathcal{O}_{t_{0}, x_{0}, t_{1}}\right.$ is the greatest cone $\mathcal{O}_{t_{0}, x_{0}, \tau}$ such that $\mathcal{O}_{t_{0}, x_{0}, \tau} \subset \Omega$.) Applying Lemma A.2 in the cone $\mathcal{O}_{t_{0}, x_{0}, t_{1}}$, we obtain:

$$
u\left(t_{0}, x_{0}\right)-v\left(t_{0}, x_{0}\right) \leq \sup _{x_{0}+B_{L\left(t_{1}-t_{0}\right)}}\left(u\left(t_{1}, .\right)-v\left(t_{1}, .\right)\right) .
$$

If $t_{1}=T$, then $u\left(t_{0}, x_{0}\right)-v\left(t_{0}, x_{0}\right) \leq \sup _{x_{0}+B_{L T}}\left(u_{T}-v_{T}\right) \leq M$, which contradicts (A.13). Hence $t_{1}<T$. We consider a point $x_{1}$ of the ball $x_{0}+\bar{B}_{L\left(t_{1}-t_{0}\right)}$ and that reaches the maximum of $u\left(t_{1}, \cdot\right)-v\left(t_{1}, \cdot\right)$, and we obtain

$$
M<u\left(t_{0}, x_{0}\right)-v\left(t_{0}, x_{0}\right) \leq u\left(t_{1}, x_{1}\right)-v\left(t_{1}, x_{1}\right) .
$$

We re-iterate the previous argument to obtain the existence of a $t_{2}$ in $\left(t_{1}, T\right)$ corresponding to the greatest cone of the form $\mathcal{O}_{t_{1}, x_{1}, t_{2}}$ and satisfying $\mathcal{O}_{t_{1}, x_{1}, t_{2}} \subset \Omega$, and then the existence of a point $x_{2}$ in $x_{1}+\bar{B}_{L\left(t_{2}-t_{1}\right)} \subset$ $x_{0}+\bar{B}_{L\left(t_{2}-t_{0}\right)}$ such that

$$
M<u\left(t_{1}, x_{1}\right)-v\left(t_{1}, x_{1}\right) \leq u\left(t_{2}, x_{2}\right)-v\left(t_{2}, x_{2}\right),
$$

and so on. Therefore we construct an increasing sequence of times $\left(t_{k}\right)$, and a sequence of points $\left(x_{k}\right)$ s.t. all the sequence $\left(t_{k}, x_{k}\right)$ belongs to the cone $\mathcal{O}_{x_{0}}$. Because $\overline{\mathcal{O}}_{x_{0}}$ is a compact set, we can extract a convergent subsequence towards an element $\left(t^{*}, x^{*}\right)$ of $\overline{\mathcal{O}}_{x_{0}}$. Moreover,

$$
M<u\left(t^{*}, x^{*}\right)-v\left(t^{*}, x^{*}\right) .
$$

If $t^{*}=T$, we obtain a contradiction. Hence $t^{*}<T$. Now by continuity, we still must have $u(t, x)-v(t, x)>M$ in a neighborhood of $\left(t^{*}, x^{*}\right)$, for instance in a tube $\mathcal{C}$ centered at $\left(t^{*}, x^{*}\right)$ and defined by

$$
\mathcal{C}:=] t^{*}-\tau_{0}, t^{*}+\tau_{0}\left[\times\left(x^{*}+B_{L \tau_{0}}\right)\right.
$$

for a $\tau_{0}>0$ sufficiently small. In particular, for any $x \in \mathcal{C},(t, x) \notin \Gamma$. On the other hand, as soon as $t^{*}-t_{k}<\tau_{0}$, we have $\mathcal{O}_{t_{k}, x_{k}, t_{k+1}} \subset \mathcal{O}_{t_{k}, x_{k}, t^{*}} \subset \mathcal{C} \subset \mathbb{R}^{d} \backslash \Gamma \equiv \Omega$. This contradicts the fact that $t_{k+1}$ is the maximal time $\tau$ such that $\mathcal{O}_{t_{k}, x_{k}, \tau} \subset \Omega$. This concludes the proof of (A.8). 


\section{REFERENCES}

[1] J.-P. Aubin, Viability theory. Birkäuser, Boston (1991).

[2] J.-P. Aubin, Viability solutions to structured Hamilton-Jacobi equations under constraints. SIAM J. Control Optim. 49 (2011) 1881-1915.

[3] J.-P. Aubin and A. Cellina, Differential inclusions, Comprehensive Studies in Mathematics. Springer, Berlin, Heidelberg, New York, Tokyo 264 (1984).

[4] J.-P. Aubin and H. Frankowska, Set-valued analysis, Birkhäuser Boston Inc., Boston, MA. Systems and Control: Foundations and Applications 2 (1990).

[5] J.-P. Aubin and H. Frankowska, The viability kernel algorithm for computing value functions of infinite horizon optimal control problems. J. Math. Anal. Appl. 201 (1996) 555-576.

[6] M. Bardi and I. Capuzzo-Dolcetta, Optimal control and viscosity solutions of Hamilton-Jacobi-Bellman equations, Systems and Control: Foundations and Applications. Birkhäuser, Boston (1997).

[7] M. Bardi, S. Koike and P. Soravia, Pursuit-evasion games with state constraints: dynamic programming and discrete-time approximations. Discrete Contin. Dyn. Syst. 6 (2000) 361-380.

[8] G. Barles, Solutions de viscosité des équations de Hamilton-Jacobi, Springer, Paris. Math. Appl. 17 (1994).

[9] R.C. Barnard and P.R. Wolenski, The minimal time function on stratified domains. Submitted (2011).

[10] E.N. Barron, Viscosity solutions and analysis in $L^{\infty}$, in Proc. of the NATO Advanced Study Institute (1999) 1-60.

[11] E.N. Barron and H. Ishii, The bellman equation for minimizing the maximum cost. Nonlinear Anal. 13 (1989) $1067-1090$.

[12] E.N. Barron and R. Jensen, Semicontinuous viscosity solutions for Hamilton-Jacobi equations with convex Hamiltonians. Commun. Partial Differ. Equ. 15 (1990) 1713-1742.

[13] E.N. Barron and R. Jensen, Relaxation of constrained control problems. SIAM J. Control Optim. 34 (1996) $2077-2091$.

[14] O. Bokanowski, E. Cristiani and H. Zidani, An efficient data structure and accurate scheme to solve front propagation problems. J. Sci. Comput. 42 (2010) 251-273.

[15] O. Bokanowski, N. Forcadel and H. Zidani, Reachability and minimal times for state constrained nonlinear problems without any controllability assumption. SIAM J. Control Optim. 48 (2010) 4292-4316.

[16] O. Bokanowski, N. Forcadel and H. Zidani, Deterministic state constrained optimal control problems without controllability assumptions. ESAIM: COCV 17 (2011) 995-1015.

[17] O. Bokanowski, J. Zhao and H. Zidani, Binope-HJ: a $d$-dimensional C++ parallel HJ solver. http://www.ensta-paristech.fr/ zidani/BiNoPe-HJ/ (2011).

[18] I. Capuzzo-Dolcetta and P.-L. Lions, Hamilton-Jacobi equations with state constraints. Trans. Amer. Math. Soc. 318 (1990) 643-683.

[19] P. Cardaliaguet, M. Quincampoix and P. Saint-Pierre, Optimal times for constrained nonlinear control problems without local controllability. Appl. Math. Optim. 36 (1997) 21-42.

[20] P. Cardaliaguet, M. Quincampoix and P. Saint-Pierre, Numerical schemes for discontinuous value function of optimal control. Set-Valued Analysis 8 (2000) 111-126.

[21] P. Cardaliaguet, M. Quincampoix and P. Saint-Pierre, Pursuit differential games with state constraints. SIAM J. Control Optim. 39 (2000) 1615-1632 (electronic).

[22] F. Clarke, Y.S. Ledyaev, R. Stern and P. Wolenski, Nonsmooth analysis and control theory. Springer (1998).

[23] M. Crandall and P.-L. Lions, Viscosity solutions of Hamilton Jacobi equations. Bull. Amer. Math. Soc. 277 (1983) 1-42.

[24] M. Crandall, L. Evans and P.-L. Lions, Some properties of viscosity solutions of Hamilton-Jacobi equations. Trans. Amer. Math. Soc. 282 (1984) 487-502.

[25] R.J. Elliott and N.J. Kalton, The existence of value in differential games, American Mathematical Society, Providence, RI. Memoirs of the American Mathematical Society 126 (1972).

[26] H. Frankowska, Lower semicontinuous solutions of Hamilton-Jacobi-Bellman equations. SIAM J. Control Optim. 31 (1993) $257-272$.

[27] H. Frankowska and S. Plaskacz, Semicontinuous solutions of Hamilton-Jacobi-Bellman equations with degenerate state constraints. J. Math. Anal. Appl. 251 (2000) 818-838.

[28] H. Frankowska and F. Rampazzo, Relaxation of control systems under state constraints. SIAM J. Control Optim. 37 (1999) 1291-1309.

[29] H. Frankowska and R.B. Vinter, Existence of neighboring feasible trajectories: applications to dynamic programming for state-constrained optimal control problems. J. Optim. Theory Appl. 104 (2000) 21-40.

[30] H. Ishii, Uniqueness of unbounded viscosity solution of Hamilton-Jacobi equations. Indiana Univ. Math. J. 33 (1984) $721-748$.

[31] H. Ishii and S. Koike, A new formulation of state constraint problems for first-order PDEs. SIAM J. Control Optim. 34 (1996) 554-571.

[32] P. Loreti, Some properties of constrained viscosity solutions of Hamilton-Jacobi-Bellman equations. SIAM J. Control Optim. 25 (1987) 1244-1252.

[33] P. Loreti and E. Tessitore, Approximation and regularity results on constrained viscosity solutions of Hamilton-Jacobi-Bellman equations. J. Math. Systems, Estimation Control 4 (1994) 467-483.

[34] K. Margellos and J. Lygeros, Hamilton-Jacobi formulation for reach-avoid differential games. IEEE Trans. Automat. Control 56 (2011)1849-1861. 
[35] M. Motta, On nonlinear optimal control problems with state constraints. SIAM J. Control Optim. 33 (1995) $1411-1424$.

[36] M. Motta and F. Rampazzo, Multivalued dynamics on a closed domain with absorbing boundary. applications to optimal control problems with integral constraints. Nonlinear Anal. 41 (2000) 631-647.

[37] D.P. Peng, B. Merriman, S. Osher, H.K. Zhao and M.J. Kang, A PDE-based fast local level set method. J. Comput. Phys. 155 (1999) 410-438.

[38] P. Saint-Pierre, Approximation of viability kernel. Appl. Math. Optim. 29 (1994) 187-209.

[39] H.M. Soner, Optimal control with state-space constraint I. SIAM J. Control Optim. 24 (1986) 552-561.

[40] H.M. Soner, Optimal control with state-space constraint II. SIAM J. Control Optim. 24 (1986) 1110-1122.

[41] P. Soravia, Pursuit-evasion problems and viscosity solutions of Isaacs equations. SIAM J. Control Optim. 31 (1993) 604-623.

[42] P.P. Varaiya, On the existence of solutions to a differential game. SIAM J. Control 5 (1967) 153-162. 